\title{
Element-Free Galerkin Method: Convergence of the continuous and discontinuous shape functions
}

\author{
Petr Krys ${ }^{*}$ and Ted Belytschko ${ }^{\dagger}$
}

August 2, 1999

\begin{abstract}
We consider numerical solutions of second-order elliptic partial differential equations, such as Laplace's equation, or linear elasticity, in two-dimensional, non-convex domains by the element-free Galerkin method (EFG). This is a meshless method, in which the shape functions are constructed by using weight functions of compact support. For non-convex domains, two approaches to the determination of whether a node affects approximation at a particular point, a contained path criterion, and the visibility criterion. We show that for non-convex domains the visibility criterion leads to discontinuous weight functions and discontinuous shape functions. The resulting approximation is no longer conforming, and its convergence must be established by inspection of the so-called consistency term. We show that the variant of the element-free Galerkin method which uses the discontinuous shape functions is convergent, and that, in the practically important case of linear shape functions, the convergence rate is not affected by the discontinuities. The convergence of the discontinuous approximation is first established by the classical and generalized patch test. As these tests do not provide an estimate of the convergence rate, the rate of convergence in the energy norm is examined, for both the continuous and discontinuous EFG shape functions and for smooth and non-smooth solutions by a direct inspection of the error terms.
\end{abstract}

\section{Introduction}

The element-free Galerkin method (EFG) is one of the so-called meshless methods. Meshless methods have been proposed in several varieties (seeTe.g. Tan overview in Duarte [1]) as Generalized Finite Difference Method (Liszka and Orkisz [2]) ГSmoothed Particle Hydrodynamics

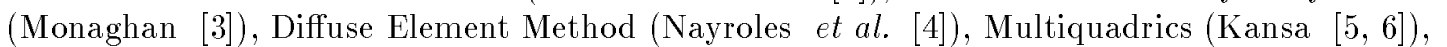
the Element-Free Galerkin Method (Belytschko et al. [7]) T Wavelet Galerkin Method (e.g.

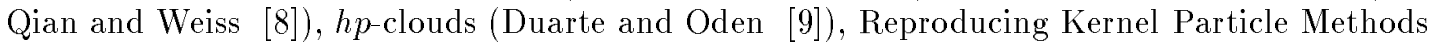
(Liu et al. [10]) Tand the Partition of Unity FEM (Babuška and Melenk [11Г12]).

Meshless methods are a rather interesting complement to traditional finite element techniques. It is possible to (i) construct arbitrarily high order approximation even for difficult fourth-order problems such as Kirchhoff-Love shells (see Krysl and Belytschko [13]) Tand (ii) the numerical integrations can be performed on an arbitrary covering of the domain so that expensive (re)meshing can be avoided; seeTe.g. TBelytschko et al. [7] for the use of background cells $\Gamma$ and Krysl and Belytschko [14] for a discussion of the background mesh. In meshless

\footnotetext{
*Research Associate, Civil Engineering, Northwestern University, Evanston, IL, USA.

tWalter P. Murphy Professor of Civil and Mechanical Engineering, Northwestern University, Evanston, IL, USA.
} 
methods 5 the discretization is based on a set of nodes (structured or scattered). The connectivity in terms of node interactions may vary with time and space $\Gamma$ and modeling of fracture $\Gamma$ free surfacesTlarge deformationsTetc. is considerably simplified.

The element-free Galerkin Method is based on a moving least squares approximation. These approximations originated in scattered data fitting $\Gamma$ where it has been studied under different names (local regression "loess" Tand moving least squares) since the 1920's; cf. Cleveland [15] and Lancaster and Šalkauskas [16]. The enforcement of essential boundary conditions in the EFG (as in all other meshless methods) requires special treatment. A number of techniques have been proposed such as point collocation L Lagrange multipliers $\Gamma$ and coupling with finite elements (seeГe.g.TBelytschko et al. [17] and Krongauz and Belytschko [18]). The coupling with finite element methods seems especially desirable as the computational costs are relatively high for the EFG method due to its dynamic connectivity; the connectivityГi.e. the interaction of nodes $\Gamma$ is not fixed by input dataTit needs to be computed. Also the shape functions are more expensive to evaluate. It is anticipated that EFG will be used primarily where needed for better accuracy and flexibility.

The goal of the present paper is to examine the construction of the shape functions in the EFG method for non-convex domains and to discuss the implications that the choices have on the convergence rates. (We call the basis functions in the approximating spaces "shape functions" as is often the case in the engineering literature on the finite element method.) The shape functions for certain constructions may be discontinuous in the immediate neighborhood of reentrant cornersTslits (cracks) and other non-convex boundaries. The method then becomes non-conforming and its convergence must be established by the second Strang lemma.

The convergence rate and the absolute accuracy depend on the construction of the shape functions at non-convex boundaries. While it is possile to construct smooth shape functions even for non-convex boundaries Tthey are rather expensive and some smooth constructions tend to deliver rather slow convergence for sharp cracks. Discontinuous shape functions are simpler to construct $\Gamma$ and yield good numerical results. Thus it is of considerable interest to establish the properties of approximations with discontinuous shape functions.

We do not attempt to present the material with mathematical rigor; ratherTwe wish to make the subject accessible to an engineer interested in applying the EFG method. Some proofs are omitted Teither because they are classical or because they can be easily found in the literature. On the other hand $\Gamma$ we attempt to make the paper self-contained and include therefore some material presented elsewhere. We establish the results for second-order elliptic equations on two-dimensional $\Gamma$ polygonal domains.

The outline of the paper is as follows: First we review the construction of the shape functions in the EFG method. We start with convex domainsTand then discuss the complications due to an introduction of a non-convex boundary. In particular $\Gamma$ both $C^{0}$ and discontinuous shape functions can be constructed in the immediate neighborhood of reentrant corners and other non-convex boundaries. Since it is known from numerical experiments that discontinuous shape functions give reasonable results at smaller cost and engender fewer complications in the construction of the EFG basis $\Gamma$ we wish to establish their convergence. For this purpose the second Strang lemma is investigated both in the classical manner by a patch test $\Gamma$ and by the generalized patch test as proposed by Stummel [19]. Both tests are shown to provide a proof of convergence $\mathrm{Cbut}$ without an indication of the convergence rate. Therefore $\Gamma$ the convergence rate is established by a direct inspection of the consistency term.

\section{Moving least squares technique}

Two approaches to the construction of the EFG basis have been proposed. The (historically) first is based on a moving least-squares approximation (used without explicit recognition in Nayroles [4] Tand later classified in Belytschko et al. [7]) Tthe second approach is an axiomatic construction based on the concept of the partition of unity; see Duarte and Oden [9] $\Gamma$ and 
Babuška and Melenk [11Г 12]. It was shown in Reference [9] that the partition-of-unity approach leads to the same set of shape functions as the former $\Gamma$ so we will use the more intuitive moving least-squares technique.

The starting point of the Element-Free Galerkin method is the following approximation $u_{h}$ of a function $u(\boldsymbol{x})$ in a small neighborhood of $\boldsymbol{x}$ by a (seemingly) polynomial expansion:

$$
u_{h}(\boldsymbol{x})=\sum_{j=1}^{n} p_{j}(\boldsymbol{x}) a_{j}(\boldsymbol{x}) .
$$

Actually the resulting approximation is more complicated; for instance $\Gamma$ it is rational when a polynomial weight function is used. The basis functions $p_{j}(\boldsymbol{x})$ are known (e.g. a complete quadratic basis in two-dimensions reads $\left.\left\{p_{j}(\boldsymbol{x})\right\}=\left\{1, x, y, x^{2}, x y, y^{2}\right\}\right) \Gamma$ the unknown coefficients $a_{j}(x)$ are solved for by the moving least-squares procedure using parameters $u_{I}$ at the nodes $\boldsymbol{x}_{I}, I=1, \ldots, M$. As is well knownT to guarantee convergence when applied to secondorder partial differential equations $\Gamma$ the approximation (1.1) should be able to reproduce a linear functionTi.e.Tit should satisfy linear consistency conditions (see e.g. Strang and Fix [20]). The polynomial basis adopted in this work is a complete polynomial of degree $k$ in the coordinates $x$ and $y$

$$
\left\{p_{j}(\boldsymbol{x})\right\}^{T}=\left\{1, \ldots x^{k}, x^{k-1} y, \ldots x y^{k-1}, y^{k}\right\}^{T} .
$$

Note that for the actual calculations $\Gamma$ the argument $\boldsymbol{x}$ should be replaced by $\overline{\boldsymbol{x}}=\boldsymbol{x}-\boldsymbol{x}^{\prime}$ to shift the origin to the evaluation point $\boldsymbol{x}^{\prime}$. Otherwise $\Gamma$ a loss of accuracy follows from the absolute values of $\boldsymbol{x}$ being too large with respect to one (the matrix $\boldsymbol{A}$ defined below is then ill-conditioned).

The moving least-squares approximation is obtained from a discrete $\Gamma$ weighted $L_{2}$ norm of the error

$$
J=\sum_{I=1}^{M} w\left(\boldsymbol{x}-\boldsymbol{x}_{I}\right)\left[p_{j}\left(\boldsymbol{x}_{I}\right) \boldsymbol{a}_{j}(\boldsymbol{x})-u_{I}\right]^{2},
$$

where $w\left(\boldsymbol{x}-\boldsymbol{x}_{I}\right)$ is a weight function of compact support (often called the domain of influence of node $I$ ). More on the weight function follows in Section 2.

This yields the following system of linear equations for the coefficients $a_{j}$ :

$$
\boldsymbol{A}(\boldsymbol{x}) \boldsymbol{a}(\boldsymbol{x})=\boldsymbol{B}(\boldsymbol{x}) \boldsymbol{u}, \quad \boldsymbol{a} \in R^{n} \Gamma \boldsymbol{u} \in R^{M}
$$

where $M$ is the number of EFG nodes whose domain of influence includes $\boldsymbol{x}$ Tand

$$
\begin{gathered}
{[\boldsymbol{A}(\boldsymbol{x})]_{i j}=\sum_{m=1}^{M} w\left(\boldsymbol{x}-\boldsymbol{x}_{m}\right) p_{i}\left(\boldsymbol{x}_{m}\right) p_{j}\left(\boldsymbol{x}_{m}\right),} \\
{[\boldsymbol{B}(\boldsymbol{x})]_{i j}=w\left(\boldsymbol{x}-\boldsymbol{x}_{j}\right) p_{i}\left(\boldsymbol{x}_{j}\right) .}
\end{gathered}
$$

Equation (1.1) can thus be put into standard form

$$
u_{h}(\boldsymbol{x})=\sum_{I=1}^{M} \phi_{I}(\boldsymbol{x}) u_{I},
$$

with $\phi_{I}(\boldsymbol{x})$ being the shape functions ${ }^{1}$

$$
\phi_{I}(\boldsymbol{x})=\sum_{j=1}^{n} p_{j}(\boldsymbol{x})\left[\boldsymbol{A}(\boldsymbol{x})^{-1} \cdot \boldsymbol{B}(\boldsymbol{x})\right]_{j I} .
$$

\footnotetext{
${ }^{1}$ The construction of the approximation in the EFG method involves an object traditionally denoted as "basis functions", i.e, the basis $p_{j}$ of the moving least-squares expression (1.1). To avoid ambiguity, we keep the name "basis function" for the $p_{j}$ 's, and we call the canonical basis functions $\phi_{I}$ of the approximating space $V_{h}$ "shape functions", as is common in the engineering literature on finite elements.
} 
Note that the EFG shape functions do not allow for the space $H_{0}^{1}(\Omega)$ (see Section 6 for notation) to be represented exactly by the EFG basis. The reason is that if constructed as outlined above the shape functions cannot be made to vanish along the boundary. The technique of coupling the EFG method with the classical finite element methods (seeTe.g. TBelytschko et al. [17] and Krongauz and Belytschko [18]) Tcan be viewed as a modification of the approximating space to account for the essential boundary conditions. In that case $\Gamma$ the space $H_{0}^{1}(\Omega)$ can be represented exactly. We assume that in the following we have modified the shape function by the coupling with finite elements along each boundary segment with Dirichlet boundary condition.

\section{Weight function}

The shape function $\phi_{I}$ is by construction compactly supportedTand its support is identical with the support of the associated weight function $w_{I}$ (see Equation (1.6)). Thus we can define a ball $B_{I}$ as the set where the weight function assumes positive values.

$$
B_{I}=\left\{\boldsymbol{x} \in R^{2}: w\left(\boldsymbol{x}, \boldsymbol{x}_{I}\right)>0\right\} .
$$

This ball is called the support of node $I \Gamma$ or the domain of influence of node $I$; the latter cognomen reflects the fact that $u_{I}$ influences the approximation of $u$ only on the support of the weigth $w_{I}$.

The weight function support can be of almost any shape; circle $\Gamma$ square $\Gamma$ rectangle etc. To beginTwe consider only the most commonly used support $B_{I}$ Ta circle of radius $r_{I}$ centered at the node $I$. We adopt weights $w_{I}$ which are positive functions of the normalized distance $r$

$$
w(r)= \begin{cases}\geq 0 & \text { for } 0 \leq r<1 \\ =0 & \text { for } r \geq 1\end{cases}
$$

with

$$
r=\frac{\left|\boldsymbol{x}-\boldsymbol{x}_{I}\right|}{r_{I}}
$$

The continuity of the resulting shape functions depends on the continuity of the weight functionTand we usually construct a sufficiently smooth weight function (e.g. $\mathrm{w} w(r)=\exp \left[r^{2} /\left(r^{2}-1\right)\right]$ for $0 \leq r<1$ Tand $w(r)=0$ for $r \geq 1$; then $\left.w(r) \in C^{\infty}\right)$. In what follows $\Gamma w e$ assume that a polynomial basis $p_{j}$ and $w \in C^{s}, s \geq 1$ are used which yields shape functions also in $C^{s}$ [21].

As will be shown laterTit is useful to modify the standard definition of the weight function (2.2) for non-convex domains to account for boundaries (cracksTreentrant cornersT and other concave parts). The resulting weight function may then be discontinuousTand also the shape functions constructed from the discontinuous weight functions are no longer even in $C^{0}$.

\section{$3 h$-Refinement for EFG}

Since there is no direct analog of a finite element in the EFG method The usual definition of the refinement parameter (or "mesh size") $h$ for finite elements is not applicable. HoweverTwe can define a useful measure of the "mesh size" $\Gamma h(x)$ Tat a point $\boldsymbol{x}$ as the diameter of the union $\mathcal{U}$ of all balls of equation (2.1) which contain point $\boldsymbol{x}$ (in the norm of (2.1)). Fixing one of the grids as the reference grid with mesh size $\widehat{h}$ Twe can define the $h$-refinement as the process $h_{j}=\widehat{h} / \sigma_{j} \Gamma$ $j=1, \ldots$ With $\sigma_{1}=1 \Gamma \sigma_{j}>\sigma_{j-1}$ for $j>1 \Gamma$ and $\lim _{j \rightarrow \infty} \sigma_{j}=\infty$. If we assume that the ratio $h_{j} / d_{j}$ Twhere $d_{j}$ is the average distance between nodes in the neighborhood of $\boldsymbol{x} \Gamma$ is held fixed during the refinement Twe obtain an EFG analog of what is understood by $h$-refinement in the finite element method. 
Since we are interested in the effect of discontinuous shape functions on the approximation around reentrant corners (crack tips) Tlet us consider this particular situation. Figure 1 shows a domain with a sharp crack. All the supports which cover the crack tip (and which define the set $\mathcal{U}$ for the crack tip) are shown. Without abandoning generality we consider the set $\mathcal{U}$ to be described in a Cartesian coordinate system $x, y$ originating at the crack tip. The $h$-refinement of $\mathcal{U}$ can then be defined by the isotropic scaling by a factor $\sigma_{j} \Gamma j \geq 1 \Gamma$ of the set $\mathcal{U}$ such that the coordinates $x_{I}, y_{I}$ of the node $I$ after the refinement are expressed by

$$
x_{I}=\widehat{x}_{I} / \sigma_{j}, \quad y_{I}=\widehat{y}_{I} / \sigma_{j},
$$

where $\widehat{x}_{I}, \widehat{y}_{I}$ are the coordinates of node $I$ in the reference gridTand $x_{I}, y_{I}$ are coordinates of the node $I$ in the refined grid. At the same timeTthe supports $r_{I}$ are scaled according to

$$
r_{I}=\widehat{r}_{I} / \sigma_{j}
$$

where $\widehat{r}_{I}$ is the radius of the support of node $I$ in the reference grid. This refinement process is illustrated in Fig. $1 \Gamma$ which shows the same domain before refinement (left) $\Gamma$ and after a refinement by $\sigma_{j}=2$.

\section{Figure 1 (scaling)}

\section{EFG approximation on non-convex domains}

Consider a scalar function $u(\boldsymbol{x})$ defined in a two-dimensional domain $\Omega$ with boundary $\partial \Omega$. The MLS technique constructs the least squares approximation locally points. The approximation $\tilde{u}(\boldsymbol{x})$ can be evaluated over the union of the nodal point supports $\Gamma$ which can extend outside the domain $\Omega \Gamma$ since $\Gamma$ in general $\Gamma$ the supports are not limited to the domain $\Omega$. We require that (i) the supports completely cover the domain and (ii) that the moment matrix $\boldsymbol{A}$ of the normal equations (1.4) is invertible at each point. However $\Gamma$ we do not restrict the supports to the domain $\Omega$. The approximation $\tilde{u}(\boldsymbol{x})$ is naturally extended to the exterior of the domain $\Omega$ by a MLS approximation defined over a union of the supports of all those nodal points whose supports overlap the domain. Such nodal points can be located anywhereTnot only outside the given domainTbut also inside other domains. Thus we have to discriminate between nodal points $\Gamma$ and to devise a way to associate the nodal points with a given evaluation point $\boldsymbol{x}$. Consider the situation in Fig. 2 (the situation may correspond $\Gamma$ for exampleTto a contact problem). The nodal points which are associated to the domain $\Omega_{1}$ are depicted as small circles. Their supports are shown as dashed circles. The $P^{t h}$ nodal point is not only located outside $\Omega_{1}$ Tbut also inside another domain $\Gamma \Omega_{2}$. In this case we do not want the nodal values of node $P$ to affect the approximation of $u$ both over $\Omega_{1}$ and over $\Omega_{2}$ Tbecause they are separate bodies.

Figure 2 (concept)

Consider another case. Figure 3 shows a situation very similar to Fig. 2. In this case $\Gamma$ however Tthere is only a single body (domain) Tand the approximation at the locations covered by the support of the $P^{t h}$ nodal point may be constructed by using the nodal value $u_{P}$ or by not using it. For instance Twe might decide to describe the approximation at the right "peninsula" by the nodal values at the nodes shown as crossesTand at the left part by nodal values at the nodes shown as circles. HoweverTthere are obviously points at which we wish both nodal sets (circles and crosses) to influence the approximationTe.g. at the point $\boldsymbol{T}$.

The question over what domains a node $P$ influences the approximation plays a crucial role in the continuity of the approximating function; in generalTwhen a discontinuity in the solution is needed (as it is the case with displacements across a crack surface) Tthe weights used in the 
MLS approximation should be discontinous. If the discontinuity is restricted to the boundary of the domain (the crack surfaces) $\Gamma$ no further consideration is required. However $\Gamma$ when the lines of discontinuity appear inside the domainTapplicability of such an approach needs to be demonstrated. That is the point we wish to investigate in what follows.

Figure 3 (onebody)

\subsection{Node inclusion criteria}

ThusTwe are led to recognize a need for a simple and consistent rule by which it can be decided whether a node $P$ affects the approximation at a given point or not. Figure 4 shows a concave corner on a domain $\Omega$. To avoid the difficult choices related to the EFG nodes being located outside the domain Twe only allow EFG nodes inside a domain or on its boundary. FurtherTwe restrict the EFG nodes to appear only in MLS approximations performed inside the domain to which they belong. Thus t wo domains cannot share a node even though such a node is located on a boundary common to both. Thus Twe consider the boundaries of domains to be perfectly "opaque" Гi.e.Tthe boundaries prevent the outside nodes to affect approximation inside.

There are (at least) two criteriaTby which to decide whether a node should be included in the MLS procedure at the location $\boldsymbol{x}$ or not. The first criterion is based on the notion of a connecting path. If two points are connected by a path lying inside the domainTsuch points can affect each other. Thus $\mathrm{T}$ we have the

1. Contained path criterion (CPC): When evaluating the MLS approximation at a point $\boldsymbol{x}$ Tinclude node $M$ only if there is a path $p$ connecting the node $M$ to the point $\boldsymbol{x} \Gamma$ which is entirely contained within an intersection of the support of the node $M$ with the domain; see the left-hand side of Fig. 4.

The second criterion is based on a visibility test $\Gamma$ and was used $\Gamma$ for exampleГin Belytschko et al. [7]. It relies on the assumption that the domain boundaries are opaque $\Gamma$ and states that a point can affect only those points of the domain which are visible from that point. Thus Twe can state the

2. Visibility criterion (VC): When evaluating the MLS approximation at a point $x$ include node $M$ only if the point $\boldsymbol{x}$ is visible from the node $M$ (in other wordsTif the straight line segment connecting $\boldsymbol{x}$ and the node $M$ does not intersect the boundary of $\Omega$ ); see the right-hand side of Fig. 4.

ThusTusing the VC for points $\boldsymbol{q}$ and $\boldsymbol{x}$ of Fig. 4 Ththe node $M$ is included in the MLS procedure for the point $q$ Tbut not for the point $\boldsymbol{x}$. On the other hand $\Gamma$ the CPC includes both points $\Gamma$ since both can be connected to the node $M$ by a path contained in the intersection of the domain $\Omega$ and of the support (dashed circle).

While the VC gives always discontinuous shape functionsT the CPC can be used to produce arbitrarily smooth shape functions. While one of the techniques constructing smooth weight functions is based on a partial transparency of the boundaries [22] $\Gamma$ the nodes are included in the shape function construction according to the CPC.

\section{Figure 4 (corner)}

The implications of the criteria are important. The second choice (VC) leads to discontinuous shape functions because the visibility test effectively cuts off the support of the weight function by the visibility ray. Thus Tin the right-hand side part of Fig. 4 T the weight function of node $M$ is non-zero in the portion of the support in the half-plane defined by the line $L$ which contains the point $q$ Tand identically zero on the line and in the half-plane which contains the point $\boldsymbol{x}$. The segment of the line $L$ between the corner tip and the support circle is thus a 
curve of discontinuity in the weight function. The discontinuous weight function subsequently generates discontinuous shape functionsTsee Equation 1.5. This is illustrated by Fig. 5Twhich shows a shape function generated by VC next to a sharp crack. Note also the pattern of discontinuity lines emanating from the crack tip in Fig. 1 for each node covering the crack tip. What is particularly important is the effect of the scaling (3.1) $\Gamma(3.2)$ through which the measure of the discontinuities diminishes uniformly with refinement.

Figure 5 (dorgan)

\subsection{Partitioning of the domain}

We consider a model problem with a single reentrant corner. The situation of two or more interacting corners is more complexTand is not considered. The MLS approximation will be constructed by the visibility criterionTso discontinuities will occur in a ray pattern around the corner as shown in Fig. 6.

Discontinuous weight functions lead to discontinuous shape functions $\phi_{I}(x)$. Let us denote the discontinuity lines by $\Gamma_{m} \Gamma$ for $m=1, \ldots, n_{d}-1$. Note that the discontinuity lines are assumed to be "short" so as to be entirely contained in the domain. In other words $\Gamma$ we assume all meshes are sufficiently fine to preclude the lines of discontinuity from intersecting the boundary of the domain except at a single point $\Gamma$ the reentrant corner. This assumption is necessary for the stability of the approximation.

The domain $\Omega$ can then be partitioned into a subdomain $\Omega_{S} \Gamma$ with boundary $\partial \Omega_{S}$ and interior $\stackrel{\circ}{\Omega}_{S}$ Twhere the shape functions are $C^{s}, s \geq 0$ Tand $n_{d}$ subdomains $\Omega_{i}$. The interiors and the boundaries of subdomains $\Omega_{i}$ are denoted $\Omega_{i} \Gamma \partial \Omega_{i}$ respectively. The boundaries $\partial \Omega_{i}$ consist partly of one or two lines of discontinuity $\Gamma_{m}$; see Fig. 6 . The subdomains $\Omega_{i}$ are adjacent to $\Omega_{S}$ across the boundaries $\hat{\partial} \Omega_{i}=\partial \Omega_{i}-\Gamma_{i}-\Gamma_{i-1} ; \Gamma_{0}\left(\Gamma_{5}\right)$ is a part of the boundary $\partial \Omega$ which is shared by $\Omega$ and $\Omega_{1}\left(\Omega_{5}\right)$. Note that the shape functions are $C^{s}$ across $\hat{\partial} \Omega_{i}, i=1, \ldots, n_{d} \Gamma$ but discontinuous across $\Gamma_{i}, i=1, \ldots, n_{d}-1$.

$$
\Omega=\bigcup_{i=1}^{n_{d}} \Omega_{i} \bigcup \Omega_{S}, \quad \text { and } \quad \stackrel{\circ}{\Omega}=\bigcup_{i=1}^{n_{d}} \stackrel{o}{\Omega}_{i} \bigcup \stackrel{\circ}{\Omega_{S}} .
$$

\section{Figure 6 (partition)}

The lengths of the discontinuity lines are bounded by the support sizes of the associated nodes as can be easily seen from Fig. 1 . Thus $\Gamma$ the measures meas $\left(\Gamma_{i}\right)$ diminish with the refinement according to (3.2). This fact becomes important laterTwhen we establish the convergence properties of the non-conforming EFG method.

\section{Common properties of the EFG and FE methods}

Much of the mathematics of the finite element methods seems to be directly applicable to the EFG method. Therefore $\mathrm{w}$ we try to exploit the commonalities of the techniques.

Let us look at the basic aspects of the finite element methodsTand let us explore how the EFG method complies with their requirements. There are three fundamental aspects which constitute the basis of the finite element technique [23]:

$\boldsymbol{F E M ~} 1$ A triangulation $\mathcal{T}_{h}$ is established over the set $\Omega \Gamma$ i.e. $\Gamma \Omega$ is subdivided into a finite number of subsets $T$ in such a way that

1. $\forall T \in \mathcal{T}_{h} \Gamma T$ is closed and its interior $\stackrel{\circ}{T}$ is non-empty and connected.

2. $\forall T \in \mathcal{T}_{h}$ Tthe boundary $\partial T$ is Lipschitz-continuous. 
3. $\bar{\Omega}=\bigcup_{T \in \mathcal{T}_{h}} T$.

4. For each distinct $T_{1}, T_{2} \in \mathcal{T}_{h}$ Tone has $\stackrel{\circ}{T_{1}} \cap \stackrel{\circ}{T_{2}}=\emptyset$.

5. Any face of a finite element $T_{1}$ is either a face of another finite element $T_{2}$ Tor it is a portion of the boundary of the set $\Omega$.

$\boldsymbol{F} \boldsymbol{E M} 2$ The spaces $P_{T}=\left\{\left.v_{h}\right|_{T} ; v_{h} \in V_{h}\right\} \Gamma T \in \mathcal{T}_{h}$ Tare complete in polynomials $\Gamma$ or at least $\Gamma$ contain functions which are "close" to being polynomials.

$\boldsymbol{F E M} 3$ The basis in the space $V_{h}$ consists of functions with finite supports.

The EFG method defines the shape functions in such a way that the requirements FEM 2 and FEM 3 are satisfied by construction. FEM 1 is not strictly necessary for the EFG method; consider for example the scheme with background cells of Belytschko et al. [7]. HoweverTthe background mesh to perform the necessary integrals of the Galerkin procedure conforms to FEM 1 exactly: the EFG approximation is formulated independent of the way the Galerkin integrals are evaluated. Then note that the requirement FEM 1.5 is designed to ensure compatibility between adjacent finite elements leading to a proper smoothness of the approximating space $V_{h}$. Thus any spatial subdivision of the domain $\Omega$ for the EFG method which complies with the points FEM 1.1 to FEM 1.4 obviates the requirement FEM 1.5.

Given the above factsTwe can conclude that the EFG method satisfies all the requirements under which convergence estimates based on interpolation theory apply to the (conforming) finite element method Tand consequently it can be expected that this FEM convergence theory will apply also to the EFG method.

\section{Convergence of non-conforming EFG method}

\subsection{Definitions}

We first list definitions of the spaces and norms used in the following. As mentioned before Twe restrict ourselves here to domains $\Omega \in R^{2}$.

- The $L^{p}(\Omega)$ spaces are defined by

$$
L^{p}(\Omega)=\left\{u \in \mathcal{M}(\Omega): \int u^{p} \mathrm{~d} x<\infty\right\},
$$

where $\mathcal{M}(\Omega)$ is a set of functions measurable in the Lebegue's sense.

- The standard Hilbert spaces $\Gamma H^{1}(\Omega)$ :

$$
H^{1}(\Omega)=\left\{u \in L^{2}(\Omega), \partial^{\alpha} u \in L^{2}(\Omega),|\alpha|=1\right\},
$$

where $\alpha$ is a multi-index; $H_{0}^{1}(\Omega)=\left\{u \in H^{1}(\Omega),\left.u\right|_{\partial \Omega}=0\right\}$.

- The norm in the space $H^{1}(\Omega)$ is defined as

$$
\|u\|^{2}=\sum_{|\alpha|=0,1}\left|\partial^{\alpha} u\right|^{2},
$$

where the seminorms $\left|\partial^{\alpha} u\right|$ are generated by

$$
\left|\partial^{\alpha} u\right|^{2}=\int_{\Omega}\left(\partial^{\alpha} u\right)^{2} \mathrm{~d} x
$$


- The Sobolev space $W^{m, p}(\Omega)=\left\{u \in L^{p}(\Omega), \partial^{\alpha} u \in L^{p}(\Omega),|\alpha| \leq m\right\}$. Note that $W^{1,2}(\Omega)=H^{1}(\Omega)$.

- The set of functions continous up to the $k^{\text {th }}$ order $\Gamma C^{k} \Gamma$ is defined as

$$
C^{k}(\Omega)=\left\{\partial^{\alpha} u \text { continuous, }|\alpha| \leq k\right\},
$$

where we have used the notation $\partial^{\alpha} u=\partial^{\alpha_{1}} \ldots \partial^{\alpha_{m}} u \Gamma \alpha=\sum_{i} \alpha_{i}$.

- The set of infinitely smooth functions with compact support $\Gamma C_{0}^{\infty}(\Omega) \Gamma$

$$
C_{0}^{\infty}(\Omega)=\left\{\partial^{\alpha} u \text { continuous with compact support, } \alpha \text { arbitrary }\right\},
$$

\subsection{Formulation of the BVP}

As a model problem $\Gamma$ we consider the following second-order boundary value problem (BVP): find $u \in H_{0}^{1}$ such that

$$
A(u, v)=F(v), \quad \forall v \in V=H_{0}^{1}(\Omega),
$$

where the bilinear form $A(.,$.$) and the linear form F($.$) are defined as (summation convention$ is adopted)

$$
\begin{aligned}
A(u, v) & =\int_{\Omega} u_{, j} v, j \mathrm{~d} x \\
F(v) & =\int_{\Omega} f v \mathrm{~d} x,
\end{aligned}
$$

and $u=0$ on the boundary $\partial \Omega$. It is assumed that $f \in L^{2}(\Omega)$. Note that the energy norm is defined in the space $H^{1}(\Omega)$ as $\|u\|^{2}=A(u, u)$.

We wish to apply the EFG approximation in the space $V_{h}$ constructed by the visibility criterion to solve the above BVP. Due to the discontinuities in the shape functions $\phi_{I} \Gamma$ the approximating space $V_{h}$ is not included in $H_{0}^{1} \Gamma$ and the bilinear form $A(.,$.$) of (6.2)$ is not defined for functions $v_{h} \in V_{h}$. HoweverTit is possible to define on $V_{h}$ an approximate bilinear form along the lines established for the non-conforming finite element method.

Using the partition of the domain $\Omega$ given in Equation (4.1) Tthe approximate bilinear form $A_{h}(.,$.$) is given by integrals over the interior \stackrel{\circ}{\Omega}_{S}$ of the subdomain $\Omega_{S} \Gamma$ and over the interiors $\stackrel{\circ}{\Omega}_{m}$ of the subdomains $\Omega_{m}$

$$
A_{h}\left(u_{h}, v_{h}\right)=\int_{\Omega_{S}} u_{h, j} v_{h, j} \mathrm{~d} x+\sum_{m=1}^{n_{d}} \int_{\Omega_{m}} u_{h, j} v_{h, j} \mathrm{~d} x,
$$

The non-conforming approximation is then formulated as

$$
A_{h}\left(u_{h}, v_{h}\right)=F\left(v_{h}\right), \quad \forall v_{h} \in V_{h} \subset L^{2}(\Omega) \bigcap_{m=1}^{n_{d}} H^{1}\left(\stackrel{\circ}{\Omega}_{m}\right) \bigcap H^{1}\left(\stackrel{\circ}{\Omega}_{S}\right) .
$$

Similarly to the energy norm $\|u\|^{2}=A(u, u) \Gamma$ for $u \in H^{1}(\Omega) \Gamma$ we define the energy norm $\|\cdot\|_{h}$ for the approximate form $A_{h}(.,$.$) as \left\|u_{h}\right\|_{h}^{2}=A_{h}\left(u_{h}, u_{h}\right)$.

The convergence of the non-conforming approximation (6.5) can be established by the second Strang lemma (cf. StrangГFix [20] Tor Ciarlet [23]):

LEMMA 1 Consider a family of discrete problems whose associated bilinear forms are uniformly $V_{h}$-elliptic (see Section 6.3). Then there exist constants $C_{1}, C_{2}$ independent of subspace $V_{h}$ such that [20,23]

$$
\left\|u-u_{h}\right\|_{h} \leq C_{1} \inf _{v_{h} \in V_{h}}\left\|u-v_{h}\right\|_{h}+C_{2} \sup _{v_{h} \in V_{h}} \frac{\left|A_{h}\left(u, v_{h}\right)-F\left(v_{h}\right)\right|}{\left\|v_{h}\right\|_{h}} .
$$


The first term on the right-hand side of (6.6) is the approximability condition from the wellknown Céa's lemmaTand the second term is the consistency error term.

REMARK 1 An additional term, which could have been included in (6.6), is the error involved in the integration of the right-hand side of (6.5) (cf. Ciarlet [23])

$$
\sup _{v_{h} \in V_{h}} \frac{\left|F_{h}\left(v_{h}\right)-F\left(v_{h}\right)\right|}{\left\|v_{h}\right\|_{h}}
$$

where $F_{h}\left(v_{h}\right)$ is obtained by integrating only over the interiors $\stackrel{\circ}{\Omega}_{m}$ and $\stackrel{\circ}{\Omega}_{S}$. This term is, however, identically equal to zero in this case, since both $v_{h}$ and $f$ are by definition square integrable, and the domains differ by a set of measure zero.

\subsection{Stability condition}

For the second Strang lemma to hold it is necessary to prove that the approximate bilinear form of equation (6.4) is uniformly $V_{h}$-elliptic. This can be deduced by noting that all the subdomains $\Omega_{m}$ are adjacent to the "smooth" subdomain $\Omega_{S}$ across curves $\hat{\partial} \Omega_{m}$ Twhere there is no discontinuity in the shape functions. Therefore $\Gamma$ no "zero-energy" modes of the functions $v_{h} \in V_{h}$ are possible. (Of course $\Gamma$ we assume that the Galerkin integrals are integrated with sufficient accuracy to prevent these modes.)

The analysis of the ellipticity of $A_{h}(.,$.$) may be based \Gamma$ for example $\Gamma$ on Brenner and Scott [24]. We need to show that the approximate bilinear form is uniformly $V_{h}(\Omega)$-elliptic $\Gamma$ i.e $\Gamma$

$$
A_{h}\left(v_{h}, v_{h}\right) \geq \alpha\left\|v_{h}\right\|^{2}, \quad \forall v_{h} \in V_{h}, \forall h,
$$

with $h$ defined in Section 3. Let us consider the operator

$$
A(u, v)=\int_{\Omega}(u, j v, j+b u v) \mathrm{d} x, \quad u, v \in H^{1}(\Omega) .
$$

Given a particular form of the boundary conditions (Dirichlet $\Gamma$ Neumann or mixed) $\Gamma$ we can proceed in the demonstration of the $H^{1}(\Omega)$-ellipticity of the operator (6.9) in the same manner as for the conforming variant of the EFG approximation spacesTif the following condition holds

$$
\sum_{i=1}^{2}\left|\frac{\partial v}{\partial x_{i}}\right|_{L^{2}(\Omega)}=0 \Rightarrow v=\text { const in } \Omega,
$$

for the non-conforming approximation space. That this is indeed the case $\Gamma$ we establish by noting that the shape functions $\phi_{I}$ (and thus also the functions $v_{h}$ ) are in general $C^{1}$ across the boundaries $\widehat{\partial} \Omega_{m}$ for weight functions $w_{I} \in C^{s}, s \geq 1$ (which we assume to be used; compare with Section 2).

\subsection{Consistency condition}

Strang and Fix [20] use Lemma 1 to construct the classical form of the patch test (stability + consistency $=$ convergence). We first apply the reasoning of Stummel [19] to verify the convergence of the non-conforming EFG method by the generalized patch test $\Gamma$ and we then prove that the regular patch test as formulated in Strang and Fix [20] is passed (in the limit $h \rightarrow 0$ ). Both of these tests are shown to be passed "in the limit" Ti.e. Tthe error goes to zero with the refinement of the mesh 


\section{Generalized Patch Test}

Stummel has shown in Reference [19] that the condition

$$
\lim _{h \rightarrow 0} \sup _{v_{h} \in V_{h}} \frac{\left|A_{h}\left(u, v_{h}\right)-F\left(v_{h}\right)\right|}{\left\|v_{h}\right\|_{h}}=\mathbf{0},
$$

is equivalent to the subspaces $V_{h}$ being closed and approximating the subspace $V$ in the sense that each $v \in V$ is a limit of a weakly convergent sequence $v_{h} \in V_{h}$. Stummel [19] has shown that for this to hold $\Gamma$ the limits $v$ of such sequences must be differentiable in the weak sense of the Sobolev space $H^{1}$. The weak derivatives are defined in a standard manner by using the Green's formula: for $u \in C^{1}(\Omega)$ and $v \in C_{0}^{0}(\Omega)$ the distributional derivative of $v$ in the direction $j \Gamma v, j$ is defined by

$$
\int_{\Omega} u_{, j} v \mathrm{~d} x+\int_{\Omega} v_{, j} u \mathrm{~d} x=0
$$

\subsection{General formulation}

The generalized patch test by Stummel [19] rests on the fact that a function $v \in L^{2}(\Omega)$ belongs to the Sobolev space $H^{1}(\Omega)$ Tif there exists $v_{, m} \in L^{2}(\Omega)$ such that

$$
\int_{\Omega}(\psi, m v+\psi v, m) \mathrm{d} x=0, \quad m=1,2,
$$

for all $\psi \in C_{0}^{\infty}(\Omega)$.

Given a geometrical subdivision of the domain $\Omega$ into a set of disjoint subdomains $\Omega_{t}$ Tsuch that the discontinuities in the functions $v_{h}$ are limited to the boundaries $\partial \Omega_{t} \Gamma$ the integration of (7.3) can be performed over the domains $\Omega_{t}$. The Gauss theorem can be applied to (7.3) to obtain

$$
\sum \int_{\partial \Omega_{t}} \psi v n_{m} \mathrm{~d} s=0, \quad m=1,2,
$$

where $n_{m}$ are the components of the normal to the boundary. Equation (7.4) can be viewed as a strong form of the generalized patch test. Its weak form can be obtained by requring that the left-hand side integrals vanish in the $\operatorname{limit} \operatorname{diam}\left(\Omega_{t}\right) \rightarrow 0$.

\subsection{Application of generalized patch test to EFGM}

The generalized patch test for the EFG method proves convergence of the non-conforming variant of the EFG method (in a weak sense) $\Gamma$ if for an arbitraryCfixed $\psi \in C_{0}^{\infty}(\Omega) \Gamma$ the lefthand side of (7.4) goes to zero as the grid is refined

$$
\lim _{h \rightarrow 0}\left[\sum_{i=1}^{n_{d}} \int_{\partial \Omega_{i}} \psi v_{h} n_{m} \mathrm{~d} s+\int_{\partial \Omega_{S}} \psi v_{h} n_{m} \mathrm{~d} s\right]=0 .
$$

The elements $v_{h}$ of the space $V_{h}$ can be written as $v_{h}(x)=\phi_{I}(x) V_{I}$ Twith $V_{I}$ being the nodal parameters. ObviouslyTthe continuity of the functions $v_{h}$ is governed by the continuity of the shape functions $\phi_{I}$. Note further that the integrals along those parts of the boundaries $\partial \Omega_{S}$ and $\partial \Omega_{i}$ across which the functions $v_{h}$ are continuous cancel when the boundary is traversed in both directions. The external boundaries can be ignored since they contribute zeroTand there remain only the discontinuity lines $\Gamma_{m}$. The patch test $(7.5)$ thus amounts to considering the integrals

$$
\sum_{m=1}^{n_{d}-1} \int_{\Gamma_{m}} \psi\left[v_{h}\right]_{m} n_{l} \mathrm{~d} s \quad l=1,2
$$


where $\left[v_{h}\right]_{m}$ is the jump of $v_{h}$ across $\Gamma_{m} \Gamma\left[v_{h}\right]_{m}=\left.v_{h}\right|_{\Gamma_{m}^{+}}-\left.v_{h}\right|_{\Gamma_{m}^{-}}$. Because $n_{l}$ is constant along $\Gamma_{m}$ Twe get

$$
n_{l} \int_{\Gamma_{m}} \psi\left[v_{h}\right]_{m} \mathbf{d} s=n_{l} V_{I} \int_{\Gamma_{m}} \psi\left[\phi_{I}\right]_{m} \mathrm{~d} s, \quad l=1,2 .
$$

The convergence of the non-conforming EFG approximation scheme is therefore governed by the integrals of (7.7). The absolute values of these integrals can be bounded by

$$
\left|\int_{\Gamma_{m}} \psi\left[\phi_{I}\right]_{m} \mathrm{~d} s\right| \leq \sqrt{\int_{\Gamma_{m}} \psi^{2} \mathrm{~d} s} \sqrt{\int_{\Gamma_{m}}\left[\phi_{I}\right]_{m}^{2} \mathrm{~d} s}
$$

Since $\psi$ is infinitely smoothTthe first term on the right-hand side of (7.8) can be bounded by using a Taylor expansion for $\psi$ Twhich gives

$$
\int_{\Gamma_{m}} \psi^{2} \mathrm{~d} s=O\left(\operatorname{meas}\left(\Gamma_{m}\right)\right)
$$

\section{3 $\quad$ Effect of $h$-refinement}

The measure of the union of the domains $\Omega_{m}$ is of the order $h^{2}$ for $\Omega_{m} \in R^{2}$. This can be deduced from the fact that the length of the discontinuity line is bounded by the parameter $h$ (compare with Section 3). We consider a refinement effected by an isotropic scaling of the union $\bigcup_{m=1}^{n_{d}} \Omega_{m}$; cf. Equations (3.1)Г(3.2). Isotropy guarantees the regularity of the refinement $\Gamma$ and hence allows us to establish a priori error estimates. This is equivalent to an assumption from the classical finite element theory that the finite element family is regularTwhich corresponds e.g. for linear triangles Tto an enforcement of a bound on the smallest angle. We assume for this purpose that the coordinate systems used are centered at the reentrant corners. Refer to Fig. 1 for illustration of the refinement concept. Nota bene that the discontinuity lines are getting shorter with the refined grid since they are defined on the supports of the weights. This has been also discussed in Section 4.1.

The shape functions $\phi_{I}(x)$ for a refined grid [as given by (3.1) and (3.2)] are related to the shape functions $\bar{\phi}_{I}(\bar{x})$ on the reference grid by

$$
\phi_{I}(x)=\bar{\phi}_{I}(\bar{x}) \text {. }
$$

Consequently the relevant integrals can be expressed as

$$
\left.\int_{\Gamma_{m}} \phi_{I}(s)^{2} \mathrm{~d} s=\sigma_{j}^{-2} \int_{\bar{\Gamma}_{m}} \phi_{I} \overline{(} \bar{s}\right)^{2} \mathrm{~d} \bar{s} .
$$

Noting that meas $\left(\Gamma_{m}\right)=\operatorname{meas}\left(\bar{\Gamma}_{m}\right) / \sigma_{j}$ Tthe Schwartz inequality and (7.9) give immediately for the refinement $j \rightarrow \infty$

$$
\left|\int_{\Gamma_{m}} \psi\left[\phi_{I}\right]_{m} \mathrm{~d} s\right| \leq C \sigma_{j}^{-3 / 2} \sqrt{\int_{\bar{\Gamma}_{m}}\left[\bar{\phi}_{I}\right]_{m}^{2} \mathrm{~d} \bar{s}}
$$

As the reference grid is fixed $\Gamma$ and $\bar{\phi}_{I}$ is bounded along $\bar{\Gamma}_{m} \Gamma$ the right-hand side of (7.12) is of order $O\left(\sigma_{j}^{-3 / 2}\right)$ Tand the refinement $\sigma_{j} \rightarrow \infty$ (i.e. $\Gamma h_{j} \rightarrow 0$ ) leads to convergence

$$
\lim _{j \rightarrow \infty}\left|\int_{\Gamma_{m}} \psi\left[\phi_{I}\right]_{m} \mathrm{~d} s\right|=0
$$

Hence $\Gamma$ we conclude that the generalized patch test (7.6) is passed in the limit $\sigma_{j} \rightarrow \infty$ (or $\left.h_{j} \rightarrow 0\right)$. 


\section{Classical patch test}

Let us inspect the convergence property of the consistency term also by using the classical patch test of Strang and Fix [20]. The strong form of the test consists in showing that for all $\tilde{u} \in P_{m}$ Twhere $P_{m}$ denotes all polynomials of degree at most $m$ Tthe exactly evaluated bilinear form $A(.,$.$) is identical to the approximate bilinear form A_{h}(.,$.$) . In our case \Gamma$ for second-order equations $\Gamma$ we have $m=1$ Ti.e. Tthe patch test is for linear polynomials $P_{1}$. In the terminology of linear elasticity the patch test requires that the discrete stiffness matrix should be correct in the limit of constant strain states $\Gamma$ ie. the discontinuities must have no effect for constant strain conditions.

The patch test can thus be formulated as (see Strang and Fix [20])

$$
A\left(\tilde{u}, \phi_{I}\right)=A_{h}\left(\tilde{u}, \phi_{I}\right), \quad \tilde{u} \in P_{1},
$$

orTwhen we consider the weak form of the patch test

$$
\lim _{h \rightarrow 0}\left[A\left(\tilde{u}, \phi_{I}\right)-A_{h}\left(\tilde{u}, \phi_{I}\right)\right]=0, \quad \tilde{u} \in P_{1} .
$$

Application of Green's formula to (4.1) gives for the partitioned domain

$$
\begin{aligned}
A(u, v) & =\int_{\Omega_{S}}(-\Delta u) v \mathrm{~d} x+\int_{\partial \Omega_{S}} v \frac{\partial u}{\partial n} \mathrm{~d} s \\
& +\sum_{m} \int_{\Omega_{m}}(-\Delta u) v \mathbf{d} x+\sum_{m} \int_{\partial \Omega_{m}} v \frac{\partial u}{\partial n} \mathrm{~d} s
\end{aligned}
$$

It is evident that the approximate bilinear form $A_{h}(u, v)$ is obtained from (8.3) if the line integrals are ignored. Substituting now $u=P_{1}=a x+b y+c \Gamma$ and $v=\phi_{I}$ Twe get

$$
A\left(P_{1}, \phi_{I}\right)=\alpha \int_{\partial \Omega_{S}}\left[\phi_{I}\right] \mathrm{d} s+\sum_{m} \alpha_{m} \int_{\partial \Omega_{m}}\left[\phi_{I}\right] \mathrm{d} s,
$$

where the area integrals vanish because of $\Delta P_{1}=0 \Gamma$ the constants $\alpha$ and $\alpha_{m}$ collect terms which correspond to $\partial P_{1} / \partial n=$ const Tand $\left[\phi_{I}\right]$ denotes the jump of $\phi_{I}$ across the corresponding boundary. The line integrals on the external parts of the boundary $\Gamma_{u}$ are identically equal to zero due to the homogeneous boundary conditions. The line integrals over those parts of the boundary which are shared by two subdomains cancel out when the functions $\phi_{I}$ are continuous across the boundary (the jump $\left[\phi_{I}\right]=0$ ) Tand thus the first term in (8.4) vanishes. There remain the integrals $\int_{\Gamma_{m}}\left[\phi_{I}\right] \mathrm{d} s$ Twhich should be identically zero to pass the strong form of the patch test. In general

$$
\int_{\Gamma_{m}}\left[\phi_{I}\right] \mathrm{d} s \neq 0, \quad m=1, \ldots, n_{d} .
$$

Thus we can conclude that the strong form of the patch test is not passed. HoweverTarguments similar to that in Section 7.3 enables us to verify that the weak form of the patch test (8.2) is passed since

$$
\lim _{h \rightarrow 0} \int_{\Gamma_{m}}\left[\phi_{I}\right] \mathrm{d} s=0, \quad m=1, \ldots, n_{d}
$$

\section{Convergence rates}

In the present section $\Gamma$ we would like to establish the rate of convergence of the EFG method for second-order problems in an energy norm. Let us first consider the conforming variant of 
the EFG method Ti.e. Tlet us assume that the shape functions are at least $\phi_{I} \in C^{0}(\Omega)$ Twhich is the case for CPC. The second term of equation (6.6) (the consistency term) is identically equal to zero. The approximability term remains (the first term in (6.6)) Tand it may be estimated by a traditional bound based on interpolation in the approximating space. The convergence rate is then given by the exponent of a mesh parameter $h$. Its exponent is dependent on the smoothness of the exact solution. We have to consider two cases:

1. The exact solution $u$ is smoothTi.e. $\Gamma u \in H^{2}$.

2. The exact solution $u$ is singular $\Gamma$ i.e. $\Gamma u \in H^{\tau} \Gamma 1<\tau<2$.

FirstTan estimate of the convergence rate will be derived for a smooth solution approximated in a space of conforming EFG basis.

\subsection{Smooth solution; Conforming EFG method}

We will use an approach similar to that developed for finite element Galerkin methods to establish the rate of convergence. In the finite element method Tone of the often used approaches to establish a rate of convergence in energy is based on interpolation theory; see Ciarlet [23] for details. To be able to use this methodology Twe use a generalization of the usual finite element interpolation based on polynomial-preserving operators [23]. (If the operator constructed in the proof is an interpolation operatorT the usual theory applies directly. The operator formulated below for EFG is in general not an interpolation operatorThowever.)

\subsubsection{Error estimate based on a polynomial-preserving operator}

We begin by establishing the approximation properties in a ball. We consider a ball $B_{J}$ which is associated with the node $J$. We construct an interpolation over the ball $B_{J}$ Tand we estimate the error of this interpolation in terms of the dimensions of the ball.

The fundamental statement is due to Ciarlet [23]. The proof is based on polynomial invariance (so as to be able to use derivative-based norms) Tand on norms of an affine mapping

$$
F(\hat{\boldsymbol{x}})=B \hat{\boldsymbol{x}}+\boldsymbol{b} \in R^{2}
$$

which is unique and invertible $\Gamma$ and maps the open set $\widehat{\Sigma}$ to the open set $\Sigma \Gamma$ with $\widehat{\Sigma}, \Sigma \subset R^{2}$.

THEOREM 1 Assume that, for some integers $k \geq 0$ and $m \geq 0$ and some numbers $p, q \in$ $[1, \infty], W^{k+1, p}\left(\widehat{B_{J}}\right)$ and $W^{m, q}\left(\widehat{B_{J}}\right)$ are two Sobolev spaces that satisfy

$$
W^{k+1, p}\left(\widehat{B_{J}}\right) \stackrel{\text { cont }}{\complement} W^{m, q}\left(\widehat{B_{J}}\right)
$$

where $\stackrel{\text { cont }}{\complement}$ is an inclusion with continuous injection [23],

and let $\widehat{\Pi} \in \mathcal{L}\left(W^{k+1, p}\left(\widehat{B_{J}}\right) ; W^{m, q}\left(\widehat{B_{J}}\right)\right)$ be a linear mapping that satisfies

$$
\widehat{\Pi} \hat{p}=\hat{p}, \quad \hat{p} \in P_{k}\left(\widehat{B_{J}}\right) \text {. }
$$

For any open set $B_{J}$ that is affine-equivalent to the set $\widehat{B_{J}}$ in the sense of (9.1), let the mapping $\Pi_{B J}$ be defined by

$$
\left\{\Pi_{B J} v\right\}^{\wedge}=\widehat{\Pi} \hat{v}
$$

for all functions $\hat{v} \in W^{k+1, p}\left(\widehat{B_{J}}\right)$ and $v \in W^{k+1, p}\left(B_{J}\right)$ in the correspondence (9.4). Then there exists a constant $C\left(\widehat{\Pi}, \widehat{B_{J}}\right)$ such that, for all affine-equivalent sets $B_{J}$,

$$
\left|v-\Pi_{B_{J}} v\right|_{m, q, B J} \leq C\left(\widehat{\Pi}, \widehat{B_{J}}\right)\left\{\operatorname{meas}\left(B_{J}\right)\right\}^{1 / q-1 / p} \frac{h^{k+1}}{\rho^{m}}|v|_{k+1, p, B_{J}},
$$


for all $v \in W^{k+1, p}\left(B_{J}\right)$. The mesh parameters $h$ and $\rho$ are defined as

$$
h=\operatorname{diam}\left(B_{J}\right), \rho=\sup \left\{\operatorname{diam}(S) ; S \text { is a ball contained in } B_{J}\right\} .
$$

\subsubsection{Polynomial-preserving operator for the EFG method}

In order to apply Theorem 1 to our argument Twe need to construct a polynomial-preserving operator for the EFG method. We define this operator on the union $\mathcal{U}$ of all balls overlapping the given ball $B_{J}$ (we assume there is $M$ such balls). The operator $\Pi$ will be defined by

$$
\Pi v=\sum_{I} \alpha_{I} \phi_{I}, I=1, \ldots, M,
$$

where $\phi_{I}$ are the shape functions associated with the nodes $I \Gamma$ and $\alpha_{I}$ are coefficients to be determined. First $\Gamma$ note that the EFG approximation preserves polynomials of order $k$ by construction $\Gamma$ with $k$ being the degree of complete polynomials included in the basis of equation (1.1). Proofs have been given in Duarte and Oden [9] and in Liu et al. [25]; a simple alternative proof is given in Belytschko et al. [26]. Thus we can write

$$
p_{j}(\boldsymbol{x})=\sum_{I} p_{j}\left(\boldsymbol{x}_{I}\right) \phi_{I}(\boldsymbol{x}) .
$$

Note that the above equation implies that the EFG approximation is a true interpolation for all polynomial functions $p$ of degree $k$ and less.

To compute $\alpha_{I}$ one can apply either of the following strategies: (i) quasi-interpolation $($ (ii) true interpolation $\Gamma($ iii) integral projection.

Quasi-interpolation The EFG method approximates any function $u(\boldsymbol{x})$ by $u_{h}(\boldsymbol{x})=\phi_{I}(\boldsymbol{x}) u_{I} \Gamma$ where $u_{I}$ is the nodal parameter associated with node $I$. In general $\Gamma u_{I} \neq u\left(\boldsymbol{x}_{I}\right)$ and $u_{I} \neq$ $u_{h}\left(\boldsymbol{x}_{I}\right)$. However $\Gamma$ since we know that polynomials reproduced by the shape functions are interpolated $\Gamma$ we can construct the required polynomial by postulating

$$
\Pi v(\boldsymbol{x})=\phi_{I}(\boldsymbol{x}) v\left(\boldsymbol{x}_{I}\right), I, J=1, \ldots, M .
$$

which means that $\alpha_{I}=v\left(\boldsymbol{x}_{I}\right)$. This operator was proposedTe.g. Tby Liu et al. [25].

Interpolation While quasi-interpolation does not $\Gamma$ in general $\Gamma$ interpolate the given function at the nodes $\Gamma$ we can devise an interpolating operator by computing the coefficients $\alpha_{I}$ from the following system of $M$ linear equations

$$
\Pi v\left(\boldsymbol{x}_{I}\right)=\alpha_{J} \phi_{J}\left(\boldsymbol{x}_{I}\right)=v\left(\boldsymbol{x}_{I}\right), I=1, \ldots, M .
$$

which are simply interpolation conditions at the nodes of all balls of the union $\mathcal{U}$. The matrix $\phi_{J}\left(\boldsymbol{x}_{I}\right)$ may be in some degenerate cases singular. However $\Gamma$ it appears that in such cases it is not even possible to construct the shape functions in the first place $\Gamma$ and thus these special cases need not be considered here. Mathematical verification of this conjecture is to the authors' knowledge not yet available.

Integral projection Duarte and Oden [9] construct the polynomial-preserving operator by an integral projectionTand they compute the coefficients $\alpha_{I}$ from

$$
\left(v-\Pi v, \phi_{I}\right)_{\Omega}=0, I=1, \ldots, M \Gamma
$$

where

$$
(a, b)_{\Omega}=\int_{\Omega} a b \mathrm{~d} x .
$$

Regardless of the construction chosen $\Gamma$ the operator $\Pi$ preserves polynomials of degree $k$ and less $\Gamma$ and linear combinations of shape functions $g=\beta_{I} \phi_{I}$. (It is therefore a projection operator $\Gamma$ $\Pi(\Pi v)=\Pi v$. 


\subsubsection{Regular refinement}

We can further specialize the above theorem by choosing a regular refinement. In this refinement Twe assume that the affine mapping is isotropic (compare with Section 3)

$$
F(\hat{\boldsymbol{x}})=h \hat{\boldsymbol{x}}+\boldsymbol{b} \in R^{n},
$$

where $h$ is the refinement parameterTthe diameter of the union of all balls containing a given point. Consequently the ratio of the inscribed ball diameter is in constant proportion to $h$. In what follows we work only with spaces $W^{s, 2}(\widehat{\Omega})$. The equation $(9.5)$ can be reformulated as

$$
\left|v-\Pi_{\Omega} v\right|_{m, 2, \Omega} \leq C(\widehat{\Pi}, \widehat{\Omega}) h^{k+1-m}|v|_{k+1,2, \Omega} .
$$

\subsubsection{Global convergence estimate}

The estimate of (9.5) is local. Finite element techniques rely on the following property of the projection operator $\Pi_{h}$ to make the transition from the local convergence estimate to a global one (cf. Ciarlet [23]).

THEOREM 2 Let $v$ be any function in the space $\operatorname{dom}\left(\Pi_{h}\right)$. Then the restriction $\left.v\right|_{T}$ belongs to the spaces $\operatorname{dom}\left(\Pi_{T}\right)$, and we have

$$
\left.\left(\Pi_{h} v\right)\right|_{T}=\Pi_{T}\left(\left.v\right|_{T}\right) \quad \forall T \in \mathcal{T}_{h} .
$$

Given the result of (9.14) $\Gamma$ the following relations can be established for the seminorms (norms) used in convergence estimates for finite elements Twhere the error is computed elementwise Tand summed over all elements $T$ in the triangulation $\mathcal{T}_{h}$

$$
|v|_{m, 2, \Omega}=\left\{\sum_{T \in \mathcal{T}_{h}}\left(|v|_{m, 2, T}\right)^{2}\right\}^{1 / 2},
$$

and

$$
\left\|v-v_{h}\right\|_{1,2, \Omega}=\left\{\sum_{T \in \mathcal{T}_{h}}\left(\left\|v-v_{h}\right\|_{1,2, T}\right)^{2}\right\}^{1 / 2} .
$$

Let us note that the EFG projection operator introduced above conforms to the requirement of the Theorem (9.14) by construction. Thus The same technique as in (9.15) and (9.16) can be applied to make the transition from a local convergence estimate of $(9.5)$ to a global convergence estimate defined on the domain $\Omega$. The domain is by construction covered by the balls $B_{I}$ Ti.e. $\Gamma$ $\Omega=\bigcup_{I}\left(B_{I} \cap \Omega\right)$. Thus 5 the global error estimates can be formulated for the EFG method by simply summing the local error ball-by-ball

$$
|v|_{m, 2, \Omega}=\left\{\sum_{I}\left(|v|_{m, 2, B_{I}}\right)^{2}\right\}^{1 / 2},
$$

and

$$
\left\|v-v_{h}\right\|_{1,2, \Omega}=\left\{\sum_{I}\left(\left\|v-v_{h}\right\|_{1,2, B_{I}}\right)^{2}\right\}^{1 / 2} .
$$

Note that to be able to use (9.17) and (9.18) we have to guarantee that the error on a ball diminishes with refinement. As can be seen from (9.13) $\Gamma$ this means that the radius of the ball should diminish with refinement. The refinement proposed in Section 3 satisfies the above requirement Tbecause the radii of domains of influence are scaled according to (3.2).

Alternative approaches to the transition from a local to a global error estimate have been proposed by Babuška and Melenk [11Г12] for the PUFEMTand by Liu et al. [25] for the reproducing least square kernel Galerkin method. 


\subsubsection{Rate of convergence for smooth solution}

Because we have assumed an isotropic mapping (9.12) Гwe can substitute the norm $\|\cdot\|_{1,2, \Omega}$ for the seminorm $|\cdot|_{m, 2, \Omega}$ Tand we obtain

$$
\| v-\left.v_{h}\right|_{1,2, \Omega} \leq C h^{k}|v|_{k+1,2, \Omega},
$$

where $v_{h}$ is the EFG solution.

\subsection{Singular solution; Conforming EFG method}

In the previous section $\Gamma$ we have established the rate of convergence of the EFG method for smooth solutions. In this proof The domain was assumed convex $\Gamma$ with a smooth boundary and the the right-hand side was smooth as well. In the present section $\mathrm{w}$ we construct an analogous estimate for the conforming variant of the $\mathrm{EFG}$ method $\Gamma$ with quasi-uniform grids $\Gamma$ and smooth right-hand side term. However Tthe domain is now expected to generate singular solutions $\Gamma$ because we allow for non-convex $\Gamma$ polygonal domains. Elliptic problems with nonconvex boundaries have been studied in depthTe.g. Tby Grisvard [27]. Earlier works are Babuška

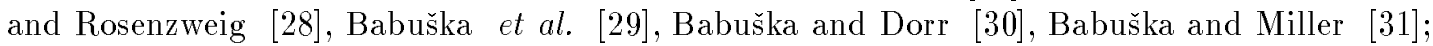
also see Bourlard et al. [32] Tand an overview article by Wahlbin [33].

We consider the Laplace's equation (6.1) with homogeneous essential boundary conditions on a two-dimensional polygonal domain. (More general operators $\Gamma$ and NeumannTor mixed boundary conditions have been treated Te.g. Tin References [29Г32].) We assume that the EFG

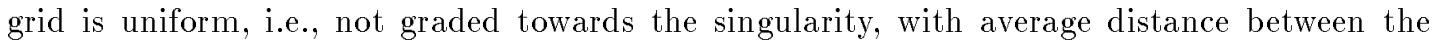
nodes being $h$. Let us denote by $\alpha_{j}$ the interior angle at each vertex of the boundary polygon. The smallest ratio

$$
\tau=\frac{\pi}{\alpha_{s}}=\min _{j} \frac{\pi}{\alpha_{j}} .
$$

indicates a singular contribution to the usual smooth part of the solutionГif $\tau<1$. The corner $v_{s}$ is associated with a singularity can be approximated by $\widetilde{u}(r, \theta)$ in polar coordinates $r$ and $\theta$ as

$$
u(r, \theta) \approx \widetilde{u}(r, \theta)=a r^{\tau} \sin (\tau \theta)+\ldots .
$$

The solution exhibits an infinite slope towards the corner. Consequently desired smoothness required by Theorem 1 Tand the estimate (9.19) no longer holds. HoweverTas shown in the references above Tthe estimate (9.19) can be generalized for less smooth solutions. Following Babuška and Rosenzweig [28] Гwe define a weighted Sobolev space by

$$
\|w\|_{W_{2, \beta}^{k}(\Omega)}^{2}=\sum_{s=0}^{k}\|w\|_{L_{2, \beta}^{s}}^{2},
$$

where

$$
\|w\|_{L_{2, \beta}^{s}}^{2}=\sum_{|i|=s} \int_{\Omega}\left(\partial^{i} w\right)^{2} \rho^{\beta} \mathbf{d} x .
$$

In (9.23) Tthe function $\rho$ is defined as [28]

$$
\rho(x)= \begin{cases}\left\|x-x_{s}\right\| & \text { for }\left\|x-x_{s}\right\|<\bar{\rho} \Gamma \\ 1 & \text { for }\left\|x-x_{s}\right\| \geq \bar{\rho} \Gamma\end{cases}
$$

where $x_{s}$ is the location of the singular vertex $\Gamma$ and $\bar{\rho}$ is prescribed. 
Using the weighted Sobolev spaces with norms of (9.22) Tit is proven in Reference [28] that the rate of convergence is given by

$$
\left\|v-v_{h}\right\|_{W_{2, \beta}^{1}(\Omega)} \leq O\left(h^{\min (k, \mu)}\right)
$$

where $\mu=\tau+\beta / 2$. For exampleTfor a sharp crack (slit) we have $\tau=1 / 2$. ThereforeTfor $\beta=0$

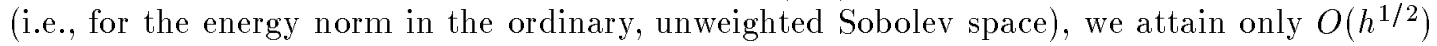
rate of convergence in energy. These results are confirmed in the numerical studies of Organ et al. [22].

Note that we again assume that the results proven in Reference [28] for finite elements apply to the EFG method without modification due to the conformance of the EFG method with the basic aspects of the FEM as discussed in Section 5. While it seems a reasonable assumption $\Gamma$ it does not constitute a rigorous proof.

REMARK 2 Note that the full convergence rate may be recovered even when not using the weighted Sobolev spaces, when the mesh is appropriately graded towards the singularity; cf. [29, 31].

\subsection{Singular solution; Non-conforming EFG method}

Here $\Gamma$ we consider again the problem of equation (6.1) on a non-convex domain Tbut the EFG method is based on discontinuous shape functions. Let us first mention certain conditions that should be met in order to arrive at useful results. First let us note that the numerical quadrature of $A_{h}(.,$.$) is assumed to be performed consistently with the partitioning (4.1) Гi.e. \Gamma$ the discontinuity lines are respected when designing the background quadrature cells in that the discontinuity lines are incorporated into the boundaries of the cells. SecondT we assume that the error due to numerical quadrature is negligible.

We would like to show that under these conditions the convergence rate is governed by the first term in (6.6). In other words $\Gamma$ we wish to establish the cost of a non-conforming approximation in terms of convergence properties. We assume a smooth exact solution $u \Gamma$ because the exponent at the mesh characteristic size $h$ in the first term of (6.6) is highest for

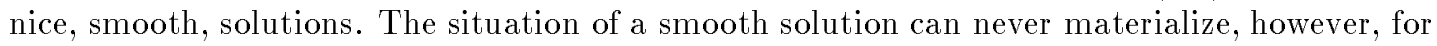
sharp re-entrant corners $\Gamma$ since such boundaries reduce the regularity of the solution.

\subsubsection{Consistency term}

Taking into account the preceding discussion $\Gamma$ we proceed next to establish the asymptotic behavior of the consistency term. We have shown in the preceding sections that the consistency term tends to zero for $h \rightarrow 0$. HoweverTneither the generalized nor the classical patch test have provided an estimate of the convergence rate.

Let us again consider the homogeneous Dirichlet problem for Laplace's equation as in Section 8 . We wish to show that the consistency term $\Gamma \mathrm{c} \Gamma$

$$
c=\sup _{v_{h} \in V_{h}} \frac{\left|A_{h}\left(u, v_{h}\right)-F\left(v_{h}\right)\right|}{\left\|v_{h}\right\|_{h}},
$$

is of order $O\left(h^{\gamma}\right)$ Twith $\gamma>0$. In that case the exponent $\gamma$ gives directly the rate of convergence in energy. If $\Gamma$ in addition $\Gamma \gamma \geq 1 \Gamma$ the rate of convergence would be governed by the first term in (6.6) $\Gamma$ and the non-conforming EFG method would be competitive in comparison to its conforming variant.

Since

$$
F\left(v_{h}\right)=A\left(u, v_{h}\right)
$$


the consistency term then becomes

$$
c=\sup _{v_{h} \in V_{h}} \frac{\left|A_{h}\left(u, v_{h}\right)-A\left(u, v_{h}\right)\right|}{\left\|v_{h}\right\|_{h}} .
$$

Also Twe can always select the function $v_{h}$ such that $\left\|v_{h}\right\|_{h}=1$ Tbecause both $A$ and $A_{h}$ are linear operators. In that case we investigate the term

$$
c=\sup _{v_{h} \in V_{h},\left\|v_{h}\right\|_{h}=1}\left|A_{h}\left(u, v_{h}\right)-A\left(u, v_{h}\right)\right|
$$

In the same manner as in Section 8 we apply Green's theorem Tand we obtain

$$
A_{h}\left(u, v_{h}\right)-A\left(u, v_{h}\right)=\sum_{m} \int_{\Gamma_{m}}\left[v_{h}\right]_{m} \frac{\partial u}{\partial n} \mathrm{~d} s
$$

where again $\left[v_{h}\right]_{m}$ denotes the jump of the function $v_{h}$ across the $m^{\text {th }}$ discontinuity line $\Gamma_{m}$.

The task now reduces to an estimate of the order of the integrals of equation (9.30). Let us estimate the norm of the difference $A_{h}\left(u, v_{h}\right)-A\left(u, v_{h}\right)$ by the Cauchy-Schwarz inequality as

$$
\begin{aligned}
\left|A_{h}\left(u, v_{h}\right)-A\left(u, v_{h}\right)\right|^{2} & \leq \sum_{m=1, n_{d}} \int_{\Gamma}\left[v_{h}\right]_{m}^{2} \mathrm{~d} s \int_{\Gamma_{m}}\left(\frac{\partial u}{\partial n}\right)^{2} \mathrm{~d} s \\
& \leq \sum_{m=1, n_{d}}\left|v_{h}\right|_{L^{2}(\Gamma)}^{2}\left|\frac{\partial u}{\partial n}\right|_{L^{2}(\Gamma)}^{2}
\end{aligned}
$$

Both $v_{h}$ and $\partial u / \partial n$ are square-integrable on $\Gamma_{m}$ by definition $\Gamma$ and we can proceed by using the Poincaré's inequality (seeTe.g.TLions and Dautray [34])

$$
|v|_{L^{2}(\Gamma)}^{2} \leq P(\Gamma) \sum_{i=1}^{2}\left|\frac{\partial v}{\partial x_{i}}\right|_{L^{2}(\Gamma)}^{2}+\operatorname{meas}(\Gamma)[\mathcal{M}(v)]^{2},
$$

where we have introduced the mean-value operator $\mathcal{M}(v)$ such that

$$
\mathcal{M}(v)=\operatorname{meas}(\Gamma)^{-1} \int_{\Gamma} v \mathrm{~d} s,
$$

and the Poincaré's constant $P(\Gamma)$. Specializing the statement (9.32) to one-dimensional domains yields (Rektorys [35])

$$
|v|_{L^{2}(\Gamma)}^{2} \leq \frac{1}{2}[\operatorname{meas}(\Gamma)]^{2}\left|\frac{\partial v}{\partial s}\right|_{L^{2}(\Gamma)}^{2}+\operatorname{meas}(\Gamma)[\mathcal{M}(v)]^{2},
$$

Note that for the discontinuity lines meas $\left(\Gamma_{m}\right)=O(h) \Gamma$ where $h=\max _{I} r_{I} \Gamma$ i.e. $\Gamma$ the largest support radius. FurtherT since both $u$ and $v_{h}$ are square-integrable $\Gamma$ their mean-values are bounded. Applying (9.34) in (9.31) Twe obtain the estimate

$$
\left|A_{h}\left(u, v_{h}\right)-A\left(u, v_{h}\right)\right|=O(h) .
$$

\subsubsection{Rate of convergence}

Combining the estimate (9.25) (using regularTunweighted Sobolev space) with (9.35) Twe find that the energy error for uniform grids is governed by

$$
\left\|u-u_{h}\right\|_{h} \leq C_{1} h^{\min (\tau, k)}+C_{2} h,
$$


with $\tau$ being the singularity strength given by (9.20). In the sense of Remark 2 we can achieve at best the convergence rate (note that $k \geq 1$ is required for second-order equations)

$$
\left\|u-u_{h}\right\|_{h} \leq C_{1} h^{k}+C_{2} h=O(h),
$$

for properly graded meshesTi.e. for meshes refined such that the effect of the singularity is eliminated in the global error [36].

We can see that the following conclusions hold for the non-conforming EFG approximation:

1. For quasi-uniform grids $\Gamma$ the rate of convergence is governed by the singularity $\Gamma$ so that $\Gamma$ for exampleTthe sharp crack reduces the rate of convergence to $1 / 2$ Tand the consistency term does not affect the rate of convergence at all.

2. For grids properly graded towards singularities $\Gamma$ the consistency term due to the nonconformity of the EFG basis (the second term in (9.36)) governs the rate of convergence for $k>1$. Hence $\Gamma$ there is no sense in using $k>1$ in (1.1) for the non-conforming variant.

3. For the practically important case of a linear basis $\Gamma$ i.e. $\Gamma$ for $k=1 \Gamma$ the energy error is governed by the singularity due to the reentrant corners $\Gamma \tau \Gamma$ for quasi-uniform meshes. For properly graded meshes $\Gamma$ the highest possible rate of convergence is achieved $\Gamma \tau=1$. Thus Ifor $k=1$ the non-conformity of the EFG method does not produce any degradation of the solution as measured by the energy error.

\section{Conclusions}

We have examined the implications of solving second-order elliptic problems in non-convex $\Gamma$ two-dimensional domains by the element-free Galerkin method.

A classification of the criteria for inclusion of a node in the construction of a shape function has been proposed. Two criteria were described; the first is based on a visibility test $\Gamma$ the second on a contained-path test. It has been shown that the visibility test leads to discontinuous shape functions. While it is possile to construct smooth shape functions even for non-convex boundaries $\Gamma$ they are rather expensive and also they tend to deliver rather slow convergence for sharp cracks (the approximation around the crack tip is overconstrained). Discontinuous shape functions are simpler to construct $\Gamma$ and yield good numerical results. Therefore $\Gamma$ the non-conforming variant of the element-free Galerkin methodTresulting when the discontinuous shape functions are used $\Gamma$ was studied with the aim of establishing its convergence properties. We have assumed that the essential boundary conditions were taken into account exactlyTas is the case with EFG/finite element coupling on polygonal domains. Also the numerical integrations were performed exactly.

The non-conforming method was studied first by the approach proposed by Stummel [19] $\Gamma$ i.e. Tby the generalized patch test. Then the classical patch test of Strang and Fix [20] was applied. Both tests prove that the so-called consistency error tends to zero as the grid is refined $\Gamma$ and the convergence of the non-conforming EFG variant was thus established. However Tthese tests provide no indication of the convergence rate.

Therefore The convergence rate of the EFG method was established by first inspecting the approximability error. The approach of Ciarlet [23] was adoptedTand the approximability error was estimated for the conforming variant by using the properties of a polynomial-preserving operator. The effect of reentrant corners (and other non-smooth boundaries) on the convergence rate was then obtained for the conforming variant following the well-established estimate based on fractional Sobolev spaces as pioneered by Babuška and others. The consistency error was then estimated for the non-conforming EFG variant by a direct inspection of the consistency term.

The following results were obtained for the convergence rates ( $k$ is the polynomial degree of the basis): 
1. For meshes properly graded to account for the singularity the non-conformity of the EFG basis governs the rate of convergence for $k>1$. Thus $\Gamma$ only the conforming variant of the EFG should be used with higher-order basis.

2. For linear basis $(k=1)$ Tthe rate of convergence is not affected by the discontinuous shape functions. For a quasi-uniform mesh the error is governed by the singularity due to the non-convex boundaries $\Gamma$ and for properly graded meshes both the approximability the consistency term estimate the same rate of convergence $\Gamma$ equal to one.

An extension of the present investigation to three-dimensional $\Gamma$ polyhedral domains $\Gamma$ is not trivial $\Gamma$ since $\Gamma$ in addition to vertex (conical) singularities $\Gamma$ edge singularities need to be considered (possibly along curved edges); cf. Grisvard [27]. HoweverT the concept is applicable to the three-dimensional case. It is also possible to apply the same reasoning to fourth-order problems.

\section{Acknowledgments}

The support of the U.S. Army Research Office and of the Air Force Office of Scientific Research is gratefully acknowledged. Tom BlackT Greg Fasshauer and Milan Jirásek are thanked for valuable comments.

\section{References}

[1] C. A. Duarte. A review of some meshless methods to solve partial differential equations. Technical Report 95-06Г Texas Institute for Computational and Applied Mathematics $\Gamma$ AustinT1995.

[2] T. Liszka and J. Orkisz. The finite difference method at arbitrary irregular grids and its applications in applied mechanics. Computers $\mathcal{E} 3$ Structures $\Gamma 11: 83-95 \Gamma 1980$.

[3] J. J. Monaghan. An introduction to SPH. Computer Physics Communications $548: 89-96 \Gamma$ 1982 .

[4] B. NayrolesTG. TouzotTand P. Villon. Generalizing the finite element method: diffuse approximation and diffuse elements. Computational MechanicsГ10:307-318Г1992.

[5] E. J. Kansa. Multiquadrics - a scattered data approximation scheme with applications to computational fluid dynamics: I. Surface approximations and partial derivative estimates. Computers and Mathematics with ApplicationsГ19:127-145Г1990.

[6] E. J. Kansa. Multiquadrics - a scattered data approximation scheme with applications to computational fluid dynamics: II. Solutions to parabolic Thyperbolic and elliptic partial differential equations. Computers and Mathematics with Applications Г19:147-161Г1990.

[7] T. BelytschkorY. Y. LuTand L. Gu. Element-free Galerkin methods. International Journal of Numerical Methods in EngineeringГ37:229-256Г1994.

[8] S. Qian and J. Weiss. Wavelet and the numerical solution of partial differential equations. Journal of Computational Physics $106: 155-175 \Gamma 1993$.

[9] C. A. Duarte and J. T. Oden. Hp clouds - A meshless method to solve boundary-value problems. Technical Report 95-05ГTexas Institute for Computational and Applied MathematicsГAustinГ1995. 
[10] W. K. LiuГSukky JunГS. LiГJ. AdeeГ and T. Belytschko. Reproducing kernel particle methods for structural dynamics. International Journal of Numerical Methods in Engineering $Г 38: 1655-1679 \Gamma 1995$.

[11] I. Babuška and J. M. Melenk. The partition of unity finite element method. Technical

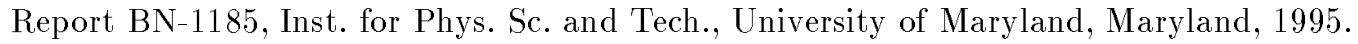

[12] I. Babuška and J. M. Melenk. The partition of unity finite element method. International Journal of Numerical Methods in Engineering, to appear $\Gamma 1996$.

[13] P. Krysl and T. Belytschko. Analysis of thin shells by the Element-Free Galerkin method. Int. J. Solids \&5 Structures $\Gamma 33: 3057-3080 Г 1996$.

[14] P. Krysl and T. Belytschko. Analysis of thin plates by the Element-Free Galerkin method. Computational MechanicsГ17:26-35Г1996.

[15] W. S. Cleveland. Visualizing data. AT\&T Bell LaboratoriesTMurray HillГN.J.T1993.

[16] P. Lancaster and K. Salkauskas. Curve and surface fitting: an introduction. Academic Press T LondonTOrlandoГ1986.

[17] T. BelytschkoTD. OrganTand Y. Krongauz. A coupled finite element-element-free Galerkin method. Computational Mechanics 17 :186-195Г1995.

[18] Y. Krongauz and T. Belytschko. Enforcement of essential boundary conditions in meshless approximations using finite elements. Computer Methods in Applied Mechanics and Engineering $\Gamma 131: 133-145 \Gamma 1996$.

[19] F. Stummel. The generalized patch test. SIAM J. Numer. AnalysisГ3:449-471Г1979.

[20] W. G. Strang and G. J. Fix. An analysis of the finite element method. Prentice-HallГ Englewood CliffsTN..J.T1973.

[21] P. Lancaster and K. Salkauskas. Surfaces generated by moving least squares methods. Mathematics of Computation $\Gamma 37: 141-158 \Gamma 1981$.

[22] D. J. OrganГM. FlemingГT. TerryГand T. Belytschko. Continuous meshless approximations for nonconvex bodies by diffraction and transparency. Computational Mechanics $\Gamma 18 \Gamma$ 1996. $225-235$.

[23] P. G. Ciarlet. Basic error estimates for elliptic problems. In Ciarlet P. G. and J. L. LionsTeditors $\Gamma$ Handbook of numerical analysis, Vol. II, Finite element methods, Part $1 \Gamma$ Amsterdam 1 1991. Elsevier TNorth-Holland.

[24] S. C. Brenner and L. R. Scott. The mathematical theory of finite element methods. SpringerVerlag $\Gamma$ New YorkT1994.

[25] W. K. LiuTS. LiTand T. Belytschko. Reproducing least square kernel Galerkin method. (i) Methodology and convergence. Computer Methods in Applied Mechanics and Engineering 1996. to appear.

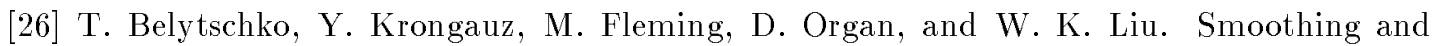
accelerated computations in the element-free Galerkin method. J. Computational and Applied Mathematics Tto appear.

[27] P. Grisvard. Elliptic problems in nonsmooth domains. Pitman Advanced Pub. ProgramГ BostonT1985. 
[28] I. Babuška and M. B. Rosenzweig. A finite element scheme for domains with corners. Numer. Math.Г20:1-21Г1972.

[29] I. BabuškaГR. B. KellogГand J. Pitkäranta. Direct and inverse error estimates for finite element with mesh refinements. Numer. Math.Г33:447-471Г1979.

[30] I. Babuška and M. R. Dorr. Error estimates for the combined $h$ and $p$ versions of the finite element method. Numer. Math.Г37:257-277Г1981.

[31] I. Babuška and K. Miller. The post-processing approach in the finite element method Part II. Calculation of stress intensity factors. International Journal of Numerical Methods in Engineering Г20:1111-1129Г1984.

[32] M. BourlardГ M. DaugeГM. S. LubumaTand S. Nicaise. Coefficients of the singularities for elliptic boundary value problems on domains with conical points. III: Finite element methods on polygonal domains. SIAM J. Numer. Anal.Г29:136-155Г1992.

[33] L. B. Wahlbin. Local behavior in finite element methods. In Ciarlet P. G. and J. L. LionsT editors $\Gamma$ Handbook of numerical analysis, Vol. II, Finite element methods, Part $1 \Gamma$ pages 353-522ГAmsterdamГ1991. ElsevierГNorth-Holland.

[34] R. Dautray and J-L. Lions. Mathematical analysis and numerical methods for science and technology, Part 2. Springer-VerlagГBerlin ; New YorkГ1988.

[35] K Rektorys. Variational methods in mathematics, science, and engineering. D. Reidel Pub. Co.TDordrechtГHolland ; BostonГU.S.А.Г1980.

[36] B. Szabo and I. Babuška. Finite element analysis. WileyГNew YorkT1991. 
Figure 1: Refinement by scaling

Figure 2: Two domains with the MLS approximation at the point $\boldsymbol{x}$

Figure 3: A single domain with the MLS approximation at the point $\boldsymbol{x}$

Figure 4: Illustration of the node inclusion criteria for a reentrant corner

Figure 5: Shape function constructed by the visibility criterion in near a crack tip

Figure 6: Partition of the domain $\Omega$ 

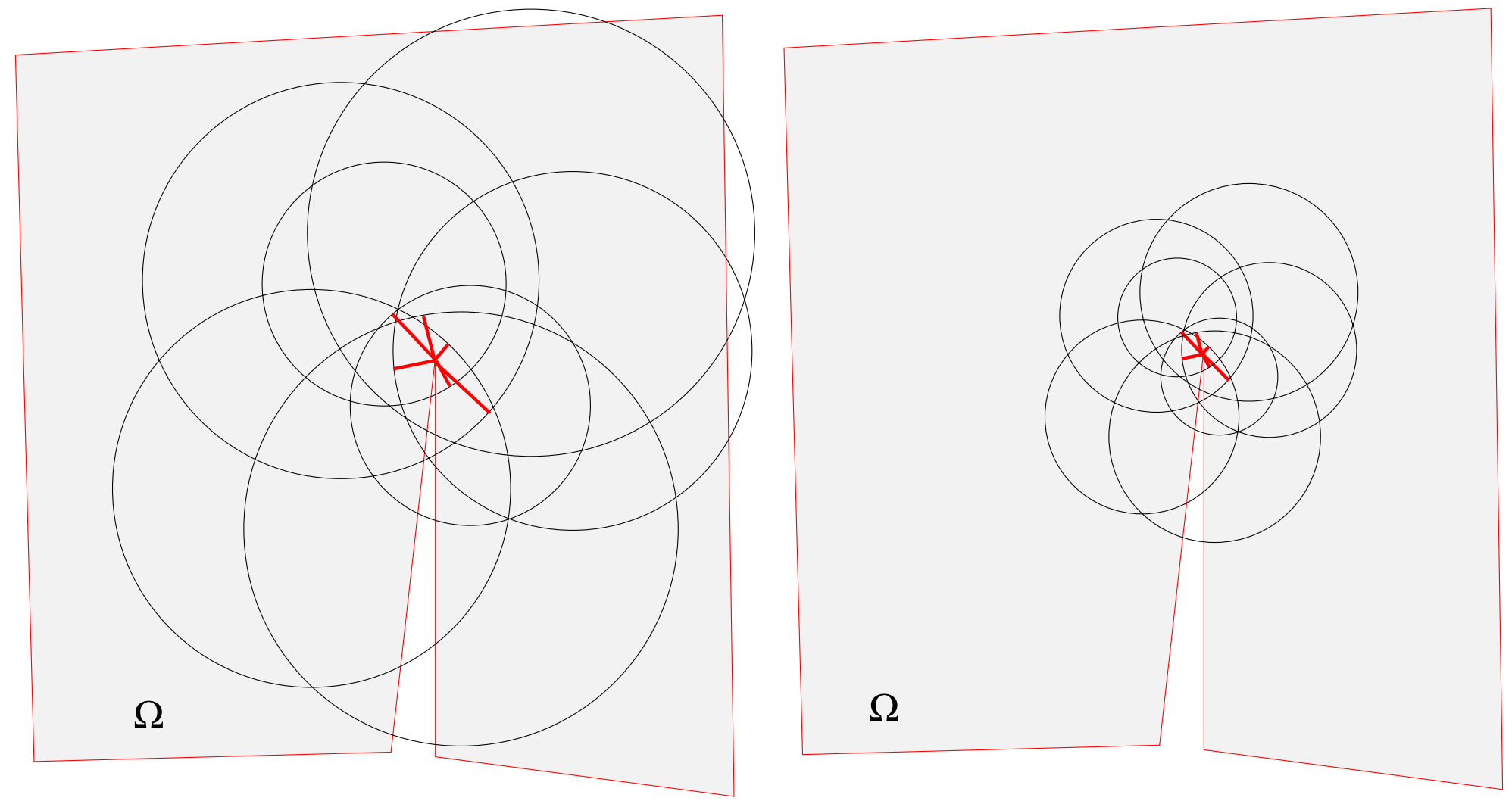

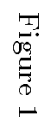




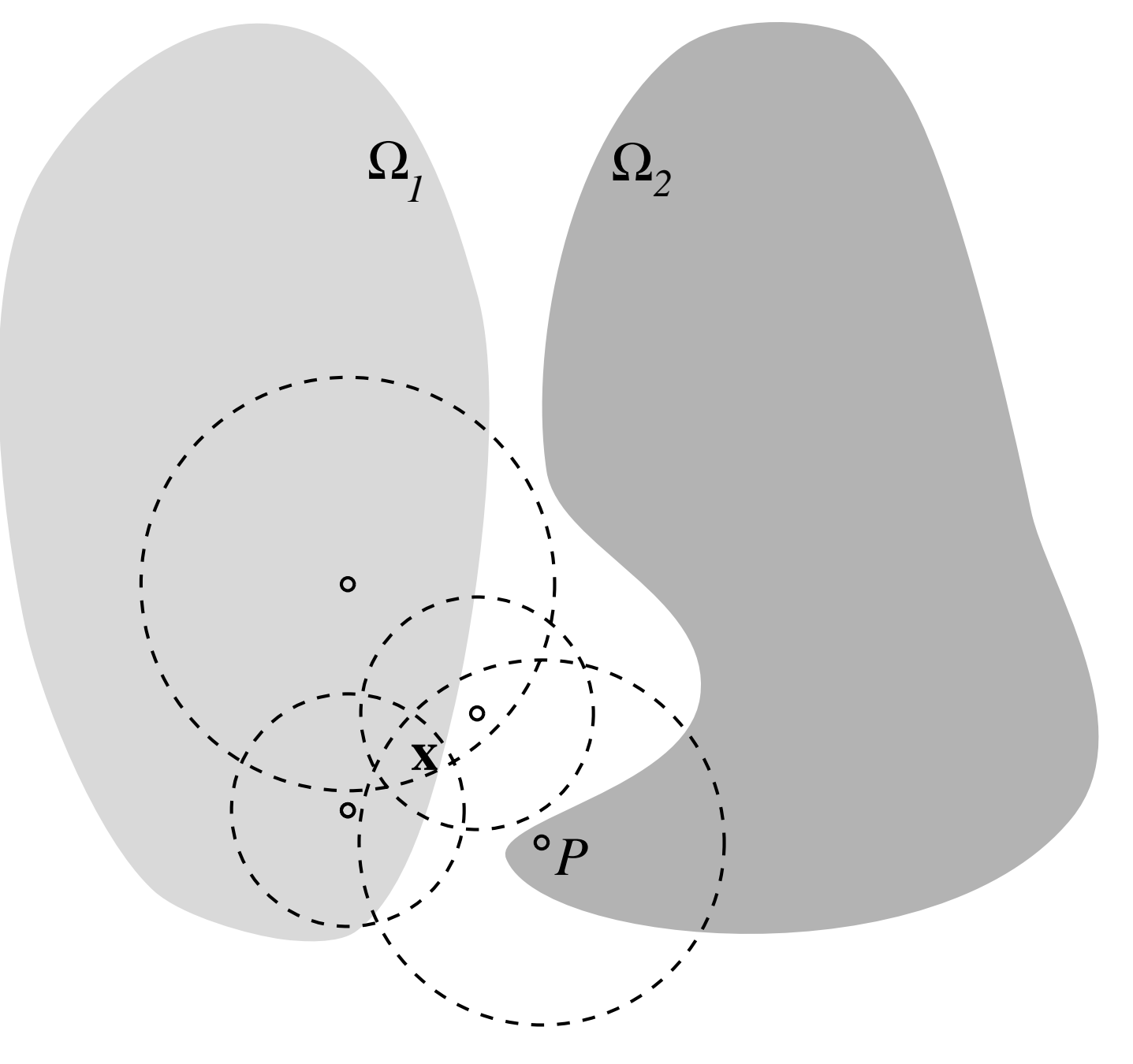

Figure 2 


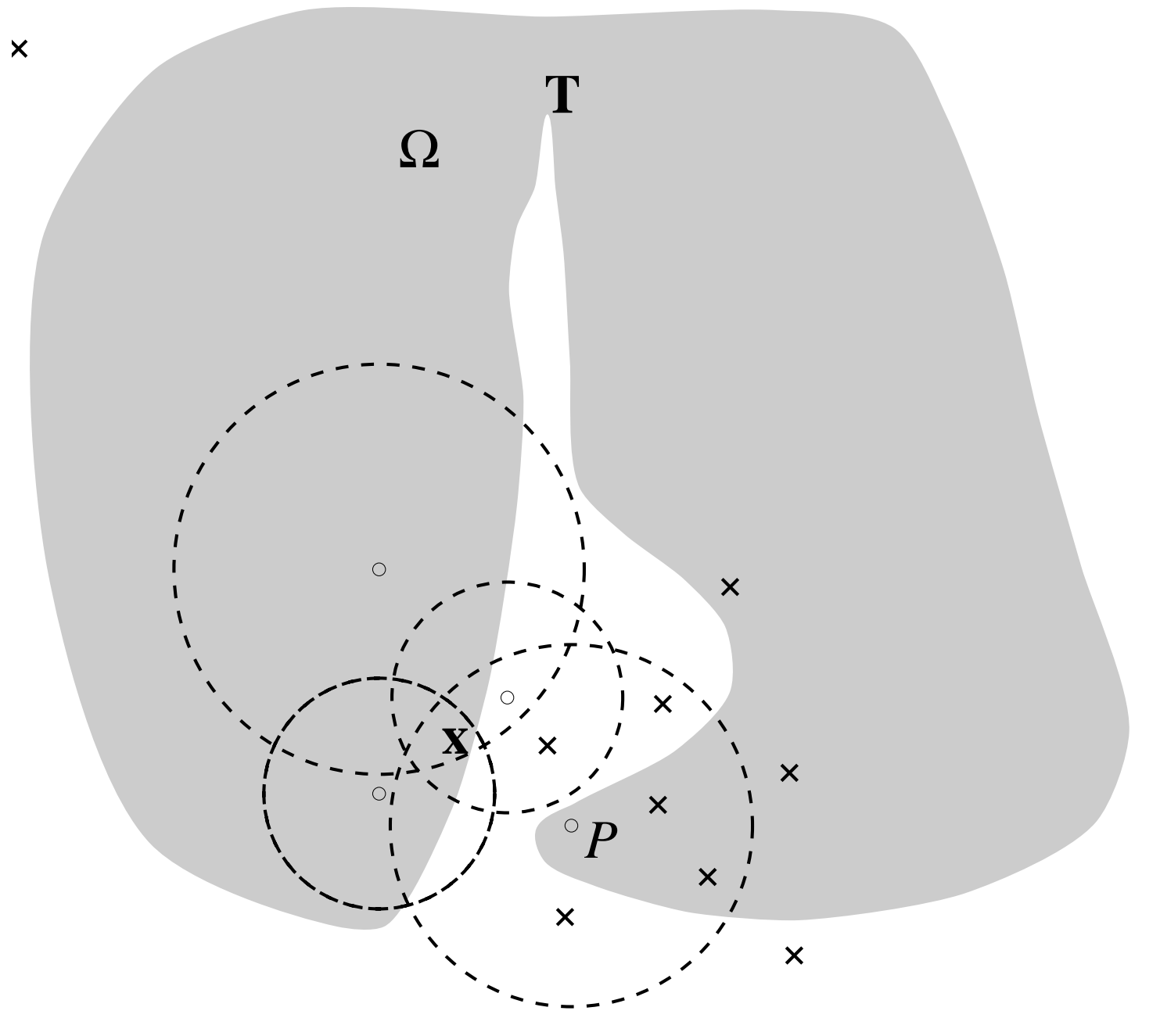

Figure 3 


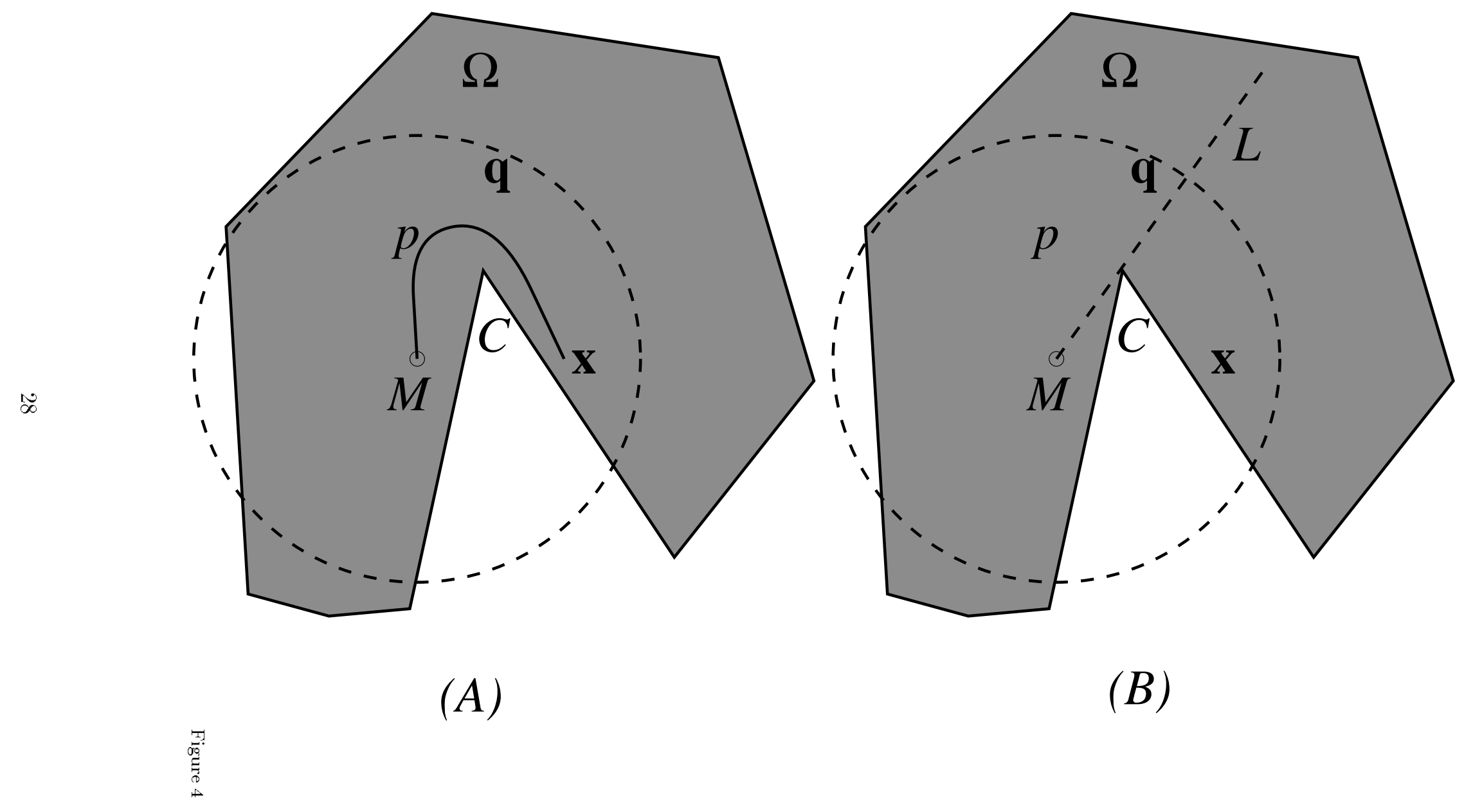




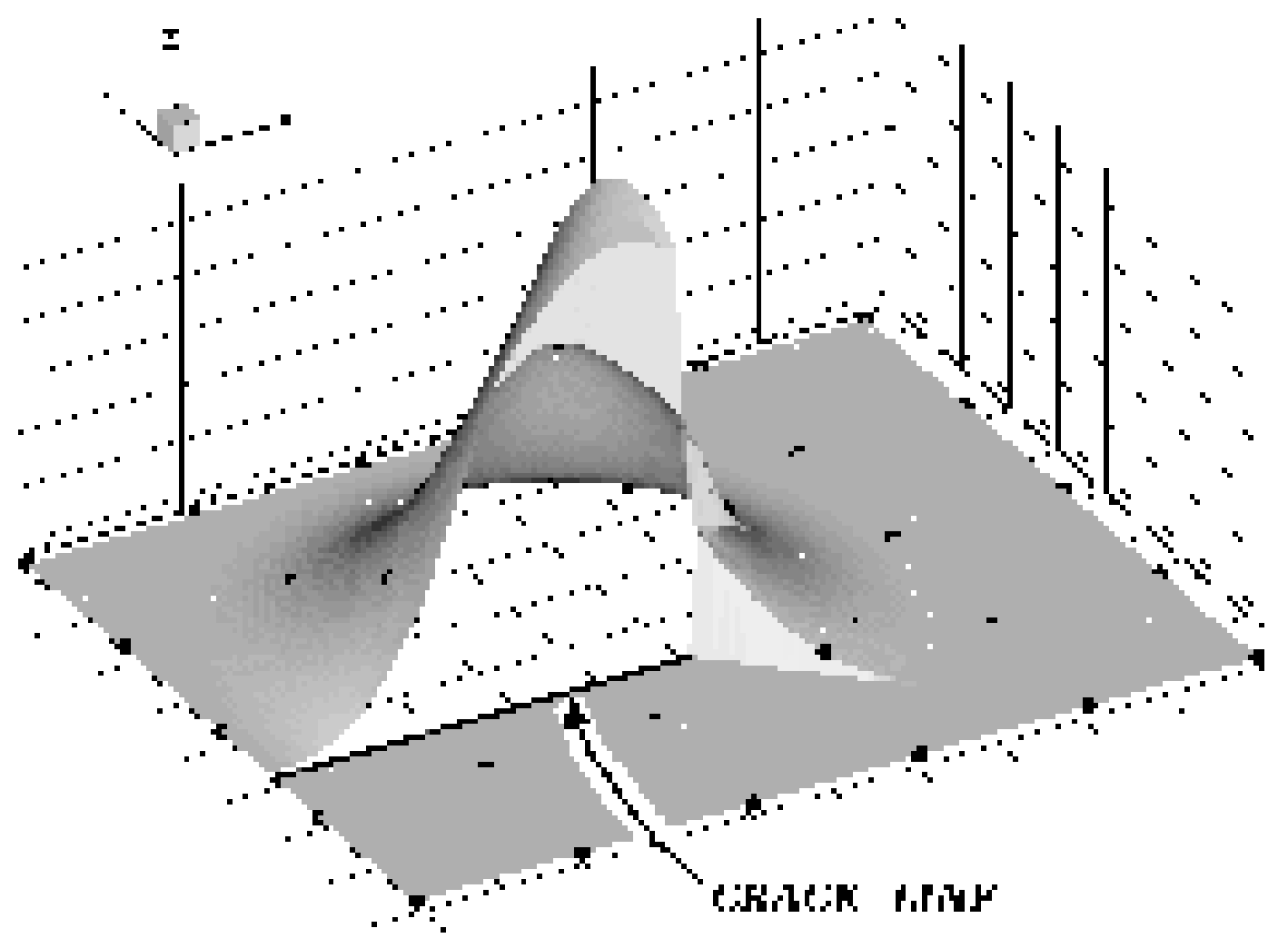

Figure 5 


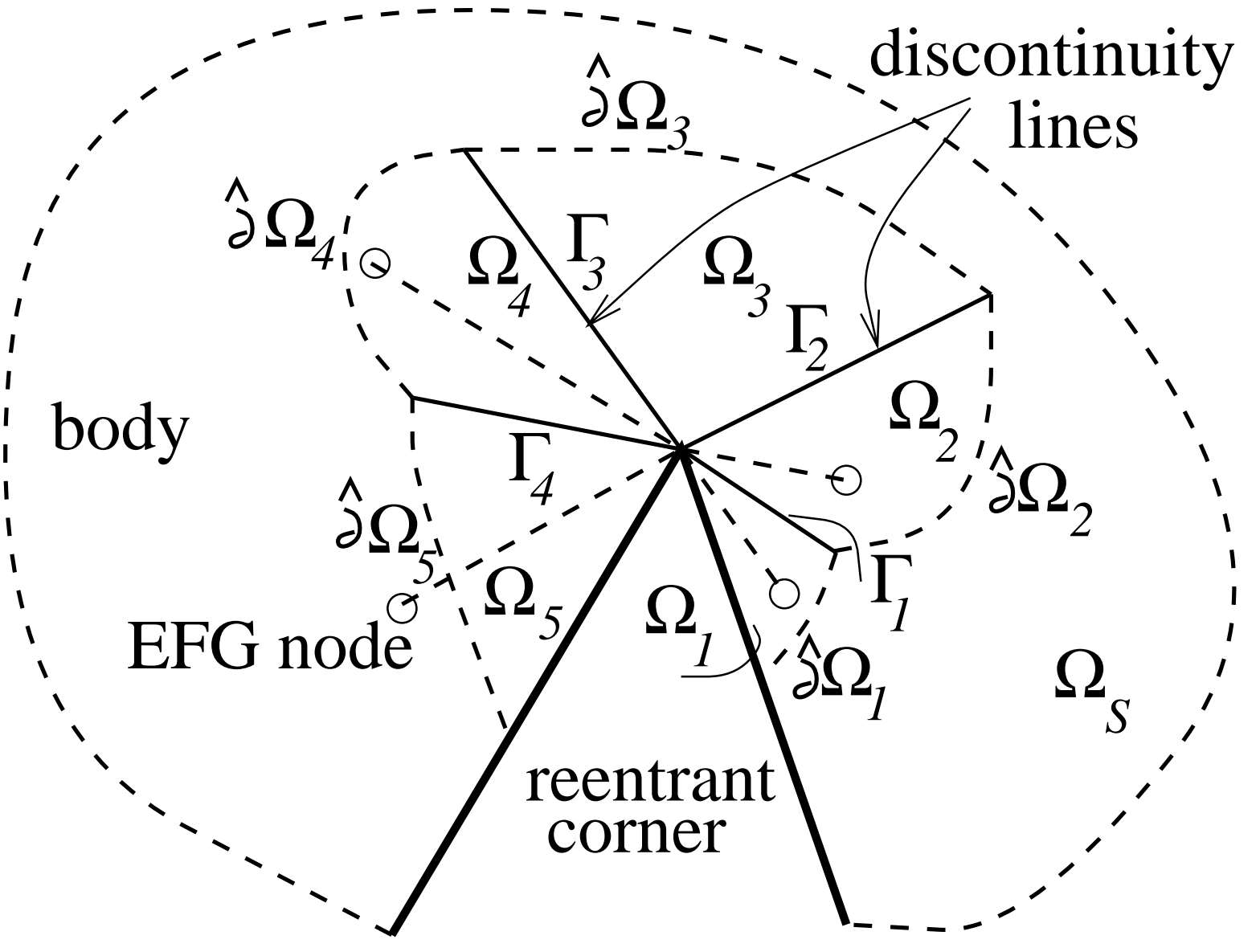

